\title{
Two Applications of Fourier's Analysis for the Interpretation of Geomagnetic Anomalies
}

\author{
Albrecht HAHN \\ Bundesanstalt für Bodenforschung, Hannover, Germany.
}

\begin{abstract}
Geomagnetic lccal anomalies measured in a horizontal plane square can be represented by a finite two-dimensional Fourier's series. The series is finite according to the finite number of information given by a measurement.

This representation of the anomalous field allows the direct calculation of the surface relief of a magnetized layer capable to produce the measured anomalies. If an average depth of the relief and the magnetization of the layer are given, the shape of the relief is unique.

A consideration of the spectrum of this representation and of the corresponding spectrum of the anomalies measured in a profile offers the possibility to give an interpretation of irregular spatial fluctuations of the anomalous field in profiles by a description of the character of the magnetization inhomogeneity in the underground. Although the anomalies are measured in profiles the magnetization distribution used for interpretation is threedimensional without a preferred direction.
\end{abstract}

\section{Introduction}

If a geomagnetic measurement comprehends only one single anomaly we usually try to interpret this result by a single magnetized body, the magnetic field of which is similar to the measured anomaly. This procedure is relatively simple. But it becomes more complicated if more than one simply shaped body is evidently responsible for the measured anomalies which anybody trying this has experienced for himself.

Unfortunately airborne measurements carried out as a help for regional geological investigations and for geophysical prospecting for oil show in most cases a variety of anomalies superposing each other to such an extent that an interpretation by a system of bodies is difficult. In order to overcome this disadvantage several methods for depth estimations have been developed whereby from the field itself, from a derivative of it or from the field reduced to the pole some characteristic quantities are taken and compared to the corresponding quantities of the magnetic field above model bodies (Vacquier et al., 1951, Giret \& Naudy, 1963). For practical reasons the variety of the shapes of those model bodies is usually restricted to vertical prisms with rectangular cross sections. But a vertical prism is, from a geological point of view, not in each case the most probable shape of the real magnetized bodies.

The magnetized bodies we are dealing with in this task of geological investigation and prospecting are assumed to be crystalline rocks showing a sufficiently high degree of metamorphism, and/or intermediary to basic igneous rocks. The complex of these rocks may be 
represented by some sort of a layer with a more or less constant magnetization and a surface relief which is responsible for the magnetic anomalies. This assumption is taken as a base for the interpretation method described in chapter 3.

In chapter 4 a method for the interpretation of the irregular spatial fluctuations of the earth's magnetic field, measured in a profile, is described. For this purpose measurements carried out in the Vogelsberg area, Germany, have been utilized. According to investigations of the rock magnetism the model for the interpretation of the field measurements is a thick horizontal layer divided into parts the magnetization of which is homogeneous within each part but is different in different parts.

From the mathematical point of view both methods start from a representation of geomagnetic anomalies by a two-dimensional finite Fourier's series. Such representations have been applied to gravimetric anomalies by Tsuboi \& Fuchida $(1937,1938)$ and to geomagnetic anomalies by Nagata (1938). Nagata, in his procedure, gives an interpretation of the measured anomalies by a thick horizontal plate with a magnetization varying in magnitude. In the method described in chapter 3 this interpretation technique is extended to a model body being a horizontal layer with homogeneous magnetization and a surface relief capable of producing the measured anomalies. The relief amplitude may reach the same order of magnitude as the average depth of the relief.

In the method described in chapter 4 the field fluctuations in a profile are characterized by their spectrum. This profile spectrum is compared to the spectrum which is to be expected in profiles measured above a model of an inhomogeneously magnetized layer. In these calculations again the two-dimensional finite Fourier's series is used. Although the field is measured only in a profile the model is a three-dimensional one without a preferred direction. But, of course, it cannot interpret any detail ; only the general character of the measured fluctuations as well as of the structure of the model are taken into account. It turns out that the continuation upward or downward of those profile spectra is dependent on the structure of the model.

\section{Physical and mathematical foundation}

\section{2.-1. General representation of anomalies by a two-dimensional Fourier's series}

Laprace's differential equation

$$
\Delta V=0
$$

written for an orthogonal coordinate system $x, y, z$ is solved by the function

$$
V=\exp \left(a x+b y+c\left(z-z_{0}\right)\right) \quad\left(z_{0} \text { being a fixed value }\right)
$$

if

$$
a^{2}+b^{2}+c^{2}=0 .
$$

Condition (3) is satisfied, e.g., by

$$
\begin{aligned}
& a=i \cdot 2 \pi k / \Lambda \\
& b=i \cdot 2 \pi \mathrm{m} / \Lambda
\end{aligned}
$$




$$
c=2 \pi \sqrt{k^{2}+m^{2}} / \Lambda
$$

whereby $k, m$ signify integers.

The solution (2) with the values (4) is reasonable from a physical point of view if the positive $z$-axis is pointing downward.

Evidently the double sum

$$
F(x, y, z)=\sum_{m=-G}^{+G} \sum_{k=-G}^{+G} K_{m}{ }^{(k)} \exp \left(2 \pi\left[\mathrm{i}(k x+m y)+\left(z-z_{0}\right) \sqrt{k^{2}+m^{2}}\right] / \Lambda\right)
$$

is also a solution of (1). $F(x, y, z)$ is, as regards the variables $x$ and $y$, a function with the period $\Lambda$.

Let a component $M$ of the earth's magnetic field be known in $r^{2}$ points of a square grid

forming a square with the surface $\Lambda^{2}$ in the plane $z=z_{0}$ (see Fig. 1), let the grid points be counted parallelly to the $x$-axis and the $y$-axis by $\rho$ and $\sigma$, respectively, and $\delta$ be the angle

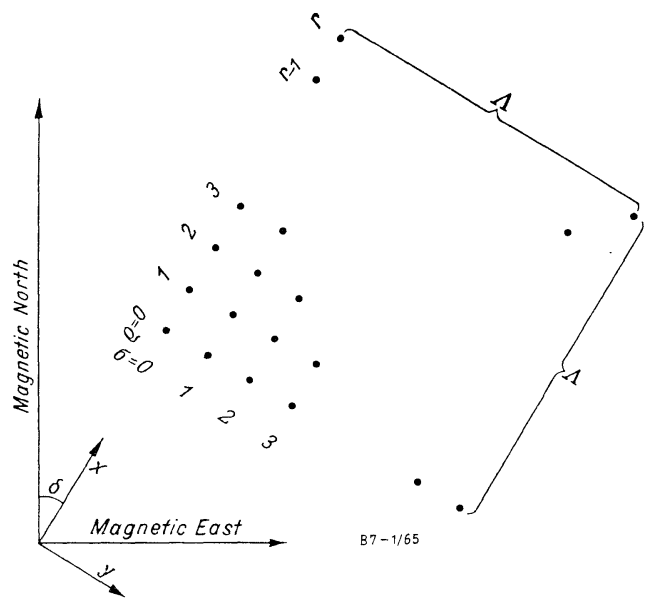

Fig. 1. Grid for taking data out of a measured area.

between the magnetic meridian and the $x$-axis ( $\delta>0$ if the $x$-axis is lying East of the magnetic meridian) then the function (5) will interpolate these $r^{2}$ values of the field component $M$ in the square if the coefficients $K_{m}{ }^{(k)}$ are determined by

$$
\begin{aligned}
& K_{m}{ }^{(k)}=\frac{1}{r^{2}} \sum_{\sigma=1}^{r} \sum_{\rho=1}^{r} M(\rho, \sigma) \exp (-2 \pi i(k \rho+m \sigma) / r) \\
& \left.\begin{array}{c}
k \\
m
\end{array}\right\}=-G, \ldots,+G ; G=\frac{r-1}{2}
\end{aligned}
$$

Moreover, the function (5) continues the given values $M$ in the whole $x, y$-plane periodically like a chessboard and gives the continuation of this entire function in $z$-direction to any level in the space where Laplace's equation is valid.

If $r$ is even, the coefficients with $k=r / 2$ and/or $m=r / 2$ must be treated in a way different from that of the other coefficients, so for convenience $r$ is chosen as an odd number.

The coefficients the indices of which have only opposite signs are conjugate complex 
quantities :

$$
K_{m}{ }^{(k)}=K_{-m}{ }^{(-k) *}
$$

Hence, in the double sum (5) the corresponding terms can be added up to real quantities (written for $z=z_{0}$ ):

$$
\begin{aligned}
& K_{m}{ }^{(k)} \exp (2 \pi i(k x+m y) / \Lambda)+K_{-m}{ }^{(-k)} \exp (-2 \pi i(k x+m y) / \Lambda) \\
& \quad=2\left[\operatorname{Re}\left(K_{m}{ }^{(k)}\right) \cos (2 \pi(k x+m y) / \Lambda)-\operatorname{Im}\left(K_{m}{ }^{(k)}\right) \sin (2 \pi(k x+m y) / \Lambda)\right] \\
& \quad=P_{m}{ }^{(k)} \cos (2 \pi(k x+m y) / \Lambda)+Q_{m}{ }^{(k)} \sin (2 \pi(k x+m y) / \Lambda)
\end{aligned}
$$

The relation between the real and the complex coefficients is evidently the following:

and

$$
\begin{aligned}
& P_{m}{ }^{(k)}=2 \cdot \operatorname{Re}\left(K_{m}{ }^{(k)}\right) \\
& Q_{m}{ }^{(k)}=-2 \cdot \operatorname{Im}\left(K_{m}{ }^{(k)}\right)
\end{aligned}
$$

$$
C_{m}{ }^{(k) 2}=P_{m}{ }^{(k) 2}+Q_{m}{ }^{(k) 2}=\left(4 K_{m}{ }^{(k)} \cdot K_{-m}{ }^{(-k)}\right)
$$

The $C_{m}{ }^{(k)}$ form the two-dimensional amplitude spectrum.

The functions (8) are distributions of the field component $M$ in the $x, y$-plane the shape of which is demonstrated in Fig. 2. They shall be called "partial field waves." The angle

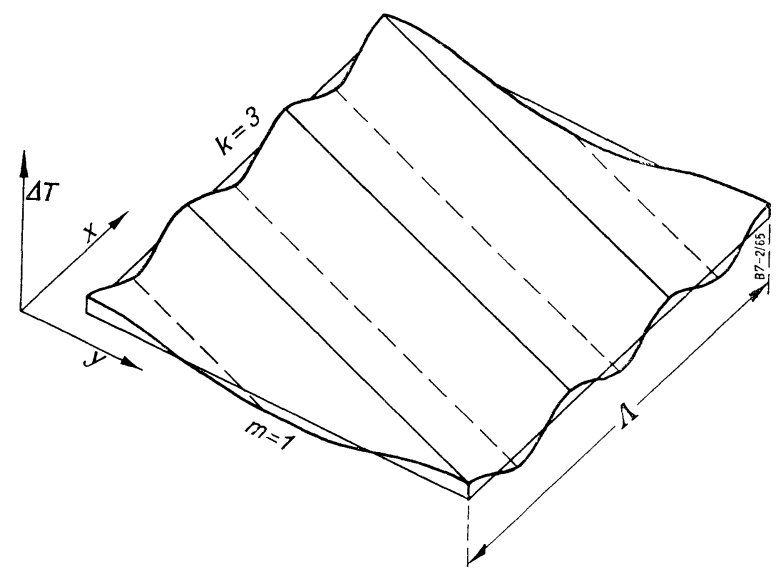

Fig. 2. The partial field wave $P_{m}{ }^{(k)} \cos (2 \pi(3 x+y) / \Lambda)$

between the direction normal to the maximum-lines of the partial field wave $k, m$ and the $x$-axis is

$$
\alpha=\tan ^{-1} \frac{m}{k}
$$

and consequently the angle between the normal direction and the magnetic meridian is

$$
\alpha+\delta
$$

(see Fig. 1)

The wavelength in normal direction is

$$
\lambda_{m}{ }^{(k)}=\frac{\Lambda}{\sqrt{k^{2}+m^{2}}}
$$

as can be seen from Fig. 2 . 


\section{2.-2. Continuation upward and downward}

According to (5) the continuation upward or downward of each partial field wave is effected by multiplying its coefficient $K_{m}{ }^{(k)}, P_{m}{ }^{(k)}, Q_{m}{ }^{(k)}, C_{m}{ }^{(k)}$ by $\exp \left(2 \pi\left(z-z_{0}\right) \sqrt{k^{2}+m^{2}} / \Lambda\right)$. The exponent is the relation of the height of continuation $z-z_{0}$ to the wavelength, multiplied by $2 \pi$. Consequently short waves are sensitive against continuation upward or downward more than long waves (see Fig. 3). The continuation is, of course, possible only in the space without magnetic sources where (1) is valid.

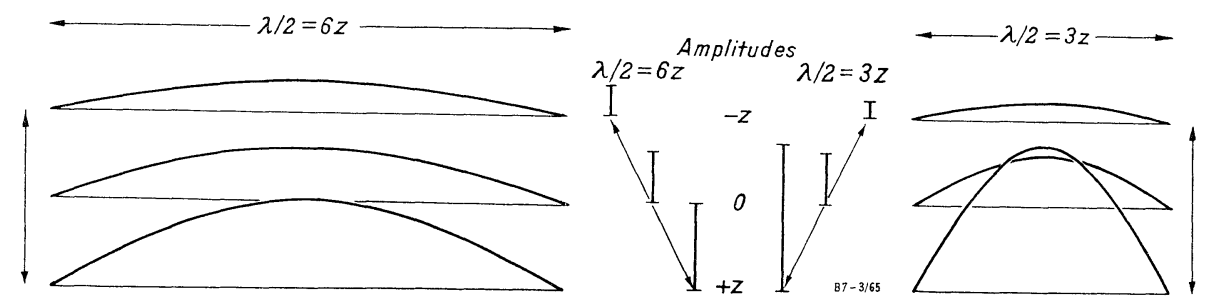

Fig. 3. Continuation upward and downward of a partial field wave. Variation of the amplitude if the height of continuation is $1 / 12$ and $1 / 6$ of the wave length, respectively.

\section{Interpretation by a surface relief}

\section{3.-1. Interpretation of one partial field wave}

The relations of the partial field waves to the corresponding magnetization waves in a horizontal plate have been deduced by Nagata (1938). They are rewritten for $\Delta T$ in the symbols of the present paper in Appendix 1. It is derived there that to the magnetization distribution

$$
J=J_{m}{ }^{(k)} \cos \left(2 \pi(k x+m y) / \Lambda-\varphi_{m}{ }^{(k)}\right)
$$

in the half space $z>\bar{z}$ the following $\Delta T$-wave in the plane $z=\bar{z}$ is corresponding:

$$
\Delta T(\bar{z}, \bar{z}, \infty)=2 \pi J_{m}{ }^{(k)}\left(\sin ^{2} I+\cos ^{2} I \cos ^{2}(\alpha+\delta)\right) \cdot \cos \left(2 \pi(k x+m y) / \Lambda-\varphi_{m}{ }^{(k)}+\pi-2 I^{\prime}\right)
$$

The amplitude of this cos-wave with the variables $x$ and $y$ is evidently

$$
|\Delta T(\bar{z}, \bar{z}, \infty)|=2 \pi J_{m}{ }^{(k)}\left(\sin ^{2} I+\cos ^{2} I \cos ^{2}(\alpha+\delta)\right)
$$

On the left hand of (14) and (15) is written the anomaly of the total intensity of the earth's magnetic field in the plane $z=\bar{z}$ which is indicated by the first symbol in the brackets. The second and the third symbol are representing the upper and the lower level, respectively, of the two plane horizontal boundaries of the volume containing the magnetization (13). The symbols on the right of (13) to (15) denote the following quantities :

$J_{m}{ }^{(k)}$ : Amplitude of the magnetization wave

$\varphi_{m}{ }^{(k)}$ : Phase angle of the $\mathrm{k}, \mathrm{m}$-magnetization wave

$I$ : Inclination of the earth's magnetic field

$I$ : Inclination of the projection of the earth's magnetic field to the normal plane of the wave

$\alpha+\delta$ : Angle between the normal plane of the wave and the magnetic meridian (see Fig. 1). 
The factor $2 \pi$ is involved here only if the emcgs-system is used. In the MKSA-system, the factor $1 / 2$ would appear instead.

From (5) it can be seen that the amplitude of the field wave in the plane

has the value

$$
z=\bar{z}-\Delta z
$$

$$
|\Delta T(\bar{z}-\Delta z, \bar{z}, \infty)|=|\Delta T(\bar{z}, \bar{z}, \infty)| \cdot \exp \left(2 \pi(-\Delta z) \sqrt{k^{2}+m^{2}} / \Lambda\right)
$$

In order to calculate the field in $z=\bar{z}$ which is produced by the plate between the planes $z=\bar{z}$ and $z=\bar{z}+\Delta z$ magnetized according to (13) this plate can be taken as the difference of the two half spaces $z>\bar{z}$ and $z>\bar{z}+\Delta z$, the latter producing a field wave with the amplitude (16) and the same phase as (14). Hence the amplitude of the field wave under consideration is

$$
\begin{aligned}
& |\Delta T(\bar{z}, \bar{z}, \bar{z}+\Delta z)|=|\Delta T(\bar{z}, \bar{z}, \infty)|\left(1-\exp \left(2 \pi(-\Delta z) \sqrt{k^{2}+m^{2}} / \Lambda\right)\right) \\
& \quad=|\Delta T(\bar{z}, \bar{z}, \infty)|\left(2 \pi(\Delta z) \sqrt{k^{2}+m^{2}} / \Lambda-\frac{1}{2 !}\left(2 \pi(\Delta z) \sqrt{k^{2}+m^{2}} / \Lambda\right)^{2}+\cdots \cdots\right)
\end{aligned}
$$

For $\Delta z \rightarrow 0$ this expression approaches

$$
\mid \Delta T\left(\bar{z}, \bar{z}, \bar{z}+\Delta z|=| \Delta T(\bar{z}, \bar{z}, \infty) \mid \cdot\left(2 \pi \sqrt{k^{2}+m^{2}} / \Lambda\right) \cdot \Delta z\right.
$$

If the thickness $\Delta z$ is small compared to the length $\Lambda / 2 \pi \sqrt{k^{2}+m^{2}}$ this thin horizontal plate with constant thickness and variable magnetization may be replaced by a plate with constant magnetization and variable thickness. This is demonstrated in Fig. 4. The upper

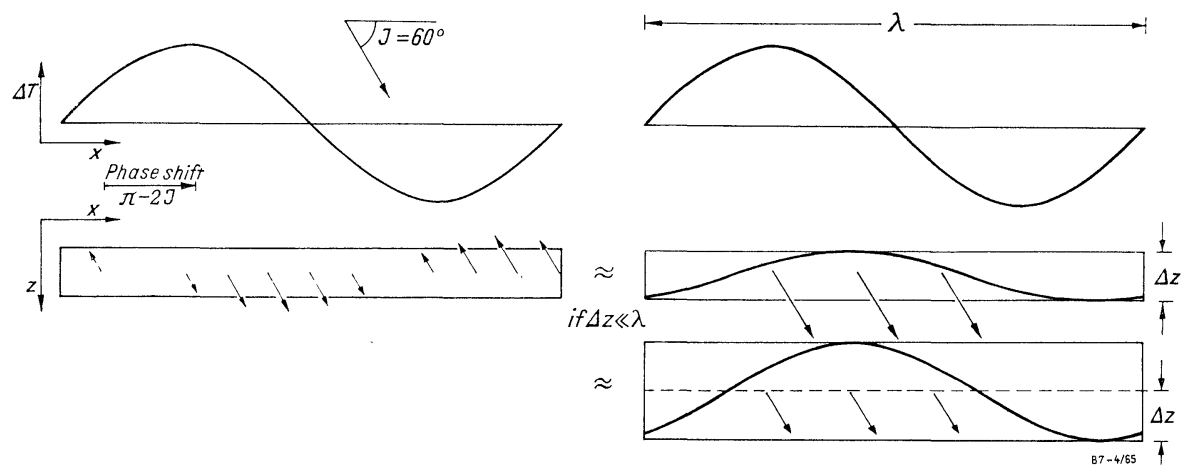

Fig. 4. The field wave plotted in the upper part of the figure can be interpreted by a plate with constant thickness and variable magnetization (left) and by plates with constant magnetization and variable thickness (right). All three models are equivalent if $\Delta z \ll \lambda$.

surface of this plate is shaped like a sin-wave. This is called "relief wave." The magnitude of the magnetization of the relief wave is equal to the double amplitude of the magnetization wave $2 J_{m}^{(k)}$, because in the magnetization wave there is a change in sign whereas the thickness of the relief wave is varying between $\mathrm{O}$ and $\Delta z$, only. Equivalent to this relief wave is, of course, the relief wave with the magnetization $J_{m}{ }^{(k)}$ and the double relief amplitude $r_{m}{ }^{(k)}=\Delta z$ instead of $\Delta z / 2$. 
Now, let

$$
\Delta T=|\Delta T(\bar{z})| \cos \left(2 \pi(k x+m y) / \Lambda-\varphi_{m}{ }^{(k)}\right)
$$

and $J$ be a field wave in the plane $z=\bar{z}$ and a fixed magnetization value, respectively. Then the corresponding relief wave has the amplitude

$$
r_{m}{ }^{(k)}=\Delta z=\frac{|\Delta T(\bar{z})|}{4 \pi^{2} J\left(\sin ^{2} I+\cos ^{2} I \cos ^{2}(\alpha+\delta)\right) \sqrt{k^{2}+m^{2}} / \Lambda}
$$

The phase of the relief wave is shifted against that of the field wave by addition of $\pi-2 I^{\prime}$ (see (13) and (14)). So the relief wave producing the field wave (19) is

$$
z=-r_{m}{ }^{(k)}\left(1+\cos \left(2 \pi(k x+m y) / \Lambda-\varphi_{m}{ }^{(k)}-\pi+2 I^{\prime}\right)\right)
$$

For an inclination between $+45^{\circ}$ and $+90^{\circ}$ this phase shift means that a maximum of the magnetization wave is lying northward of the corresponding field maximum for $\pi-2 I^{\prime}$.

The relief wave is formulated in equation (21) as it is represented in Fig. 4. The negative sign is only due to the fact that for an inclination of $+90^{\circ}$ under the maximum of the $\Delta T$ wave the highest elevation of the relief wave is situated which has the lowest $z$-value.

In order to make the incorrectness of the approximation (18) as small as practically possible, instead of (21) the relief wave

$$
z=-r_{m}{ }^{(k)} \cos \left(2 \pi(k x+m y) / \Lambda-\varphi_{m}{ }^{(k)}-\pi+2 I^{\prime}\right)
$$

is taken. Although hereby magnetic sources penetrate the plane $z=\bar{z}$, so that Laplace's equation may no longer be applied to this plane and its surrounding, the expression (22) is the best approximation to the given problem. However, this shall not be proved here.

\section{3.-2. Interpretation of the entire measured field}

The interpretation of a field measured in the plane $z=z_{0}$ is done in the following way: A square-shaped part of the field is analyzed according to (6) leading to the double sum of type (5) which consists of partial field waves counted by a pair of figures $k, m$. These partial field waves are numbered by a single figure according to some system or the other. Furthermore a starting depth of the interpretation $\bar{z}$ and a magnetization value $J$ are chosen. A horizontal plate magnetized parallel to the earth's magnetic field is supposed to have its upper plane in the depth $\bar{z}$. The lower plane is assumed at least as deep as the deepest valley of the interpretation relief will reach.

Now the first wave is continued downward to the starting depth $\bar{z}$. There it is converted to a relief wave as was described in para 3.1. In areas where the relief wave shows positive $z$-values, a valley is digged out of the presumed horizontal plate; in areas with negative $z$ values a hill of magnetized substance is placed on the surface of the plate. The second wave then has a relief in front instead of a plane. So it is continued downward in every point to the depth given by that relief and there it is converted to a point of a relief wave which is added to the first one. Hence the amplitude of this second wave will be smaller in those points where a hill of the first one is found, and bigger in valleys of the first one. In short: The second wave undergoes an amplitude modulation by the relief of the first one. 
Any following wave is modulated by the relief built up prior to its treatment. This is shown schematically in Fig. 5.

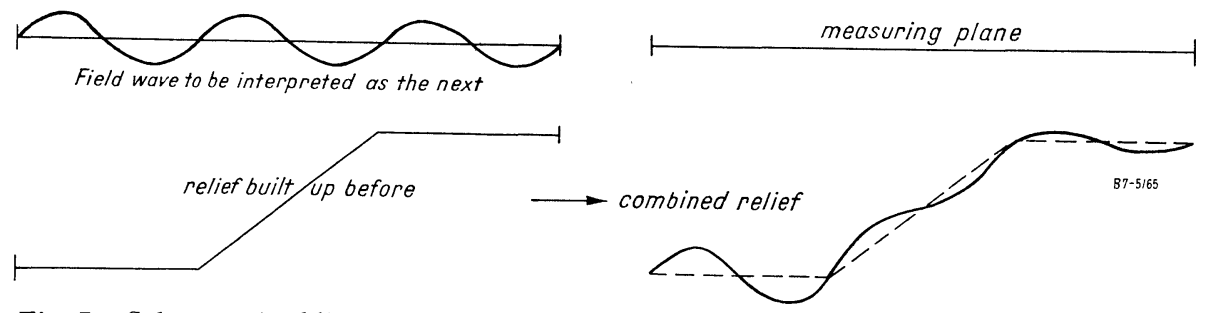

Fig. 5. Scheme of adding up the relief waves. In each place the amplitude of the relief wave is modulated according to the depth of the relief built up before.

This method of adding up is not an exact one. Each wave slightly influences its neighbouring waves. But it is the optimum method which can be found under the condition that each wave shall be treated once only. The arrangement of the sequence of the partial waves to be treated hardly affects the shape of the relief. In critical cases it was found practical to take the short waves first.

\section{3.-3. Avoiding Gibbs' phenomenon}

As mentioned in para 2.1. the interpolation function (5) continues the field inside the analysed square into the entire plane with the yeriod $A$ in $x$ and $y$. If the square is part of a geomagnetic survey map the values along opposite sides are usually more or less different. This difference would take place in the interpolation function across the boundary lines of the square within a distance equal to the grid space and would give rise to let Gibbs' phenomenon appear in the environment of the boundary lines which would be a bad interpolation. To avoid this phenomenon a smoothing stripe containing three or five rows of points is

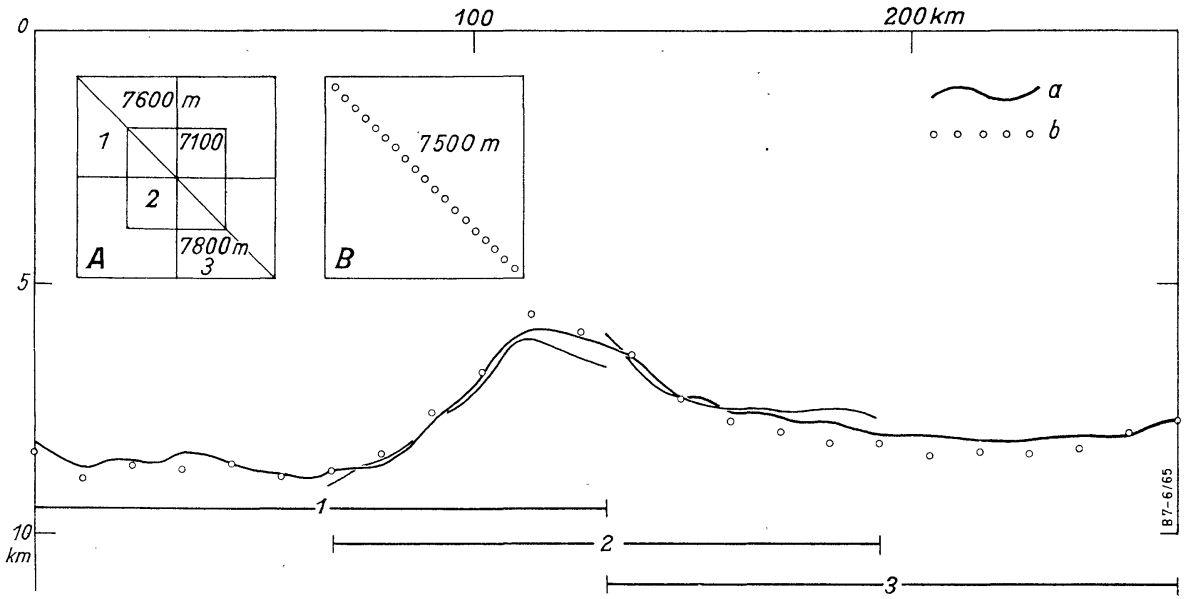

Fig. 6. Comparison of the reliefs obtained by interpretation of the three partial squares $A$ (full lines) and the relief interpreting the entire square $B$ (open circles). The starting depth values used for the reliefs are indicated in the respective squares. The horizontal and the depth scale are indicated on top and on the left side of the figure, respectively. 
covered with artificial field values providing a smooth connection of the values along opposite sides of the sqare which now are separated by an interval of four or six grid units in the periodcal interpolation function.

This method has been tested in the diagonal profile of a square obtained by relief calculation for the whole square on the one hand and by three reliefs calculated for the three partial squares situated as shown in Fig. 6 . The boundary error turned out to be sufficiently small.

\section{3.-4. A practical application}

An example for the calculation of a relief interpreting a given measured field is shown

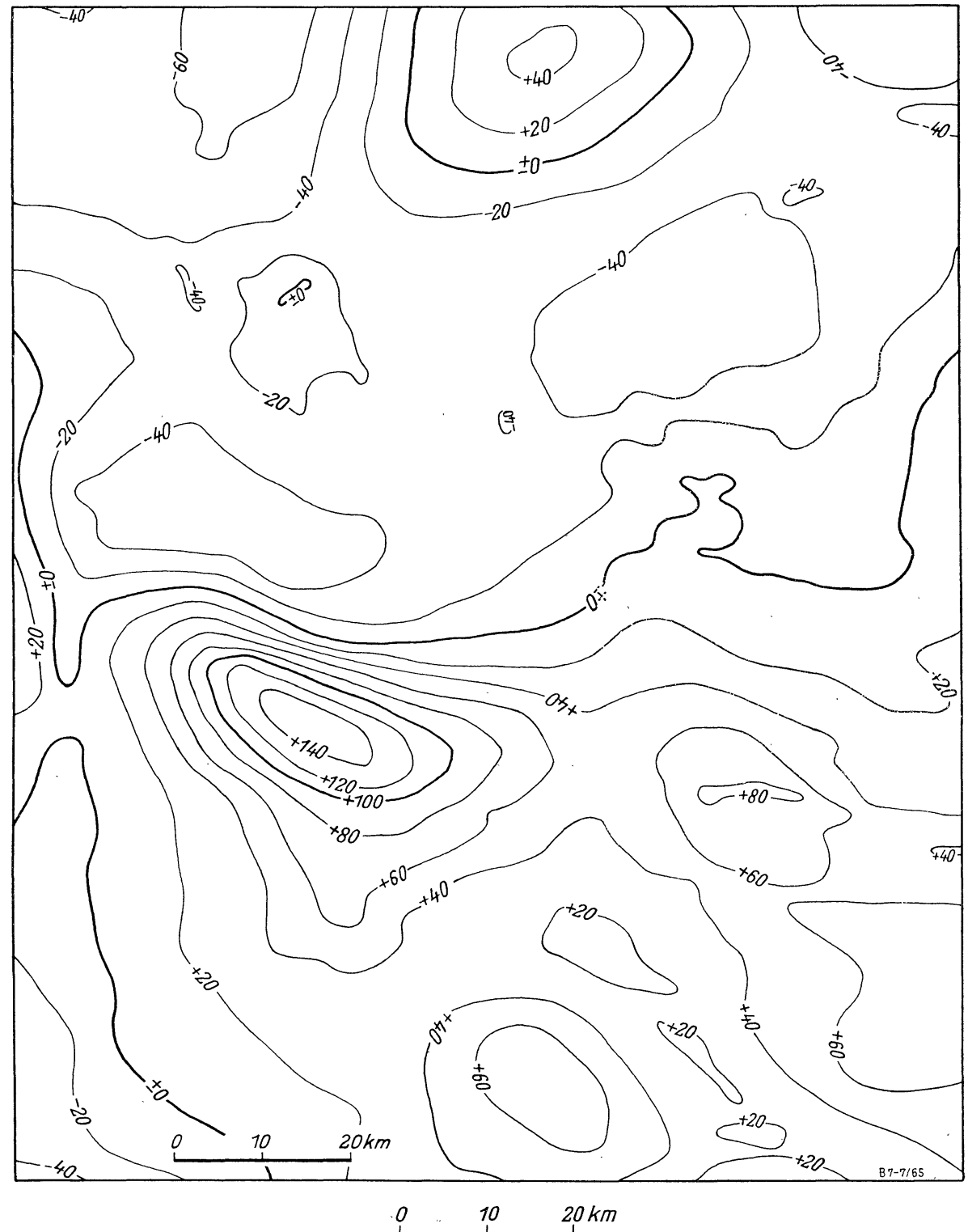

Fig. 7. Contour map of an airborne $\Delta T$-measurement in NW-Germany. 

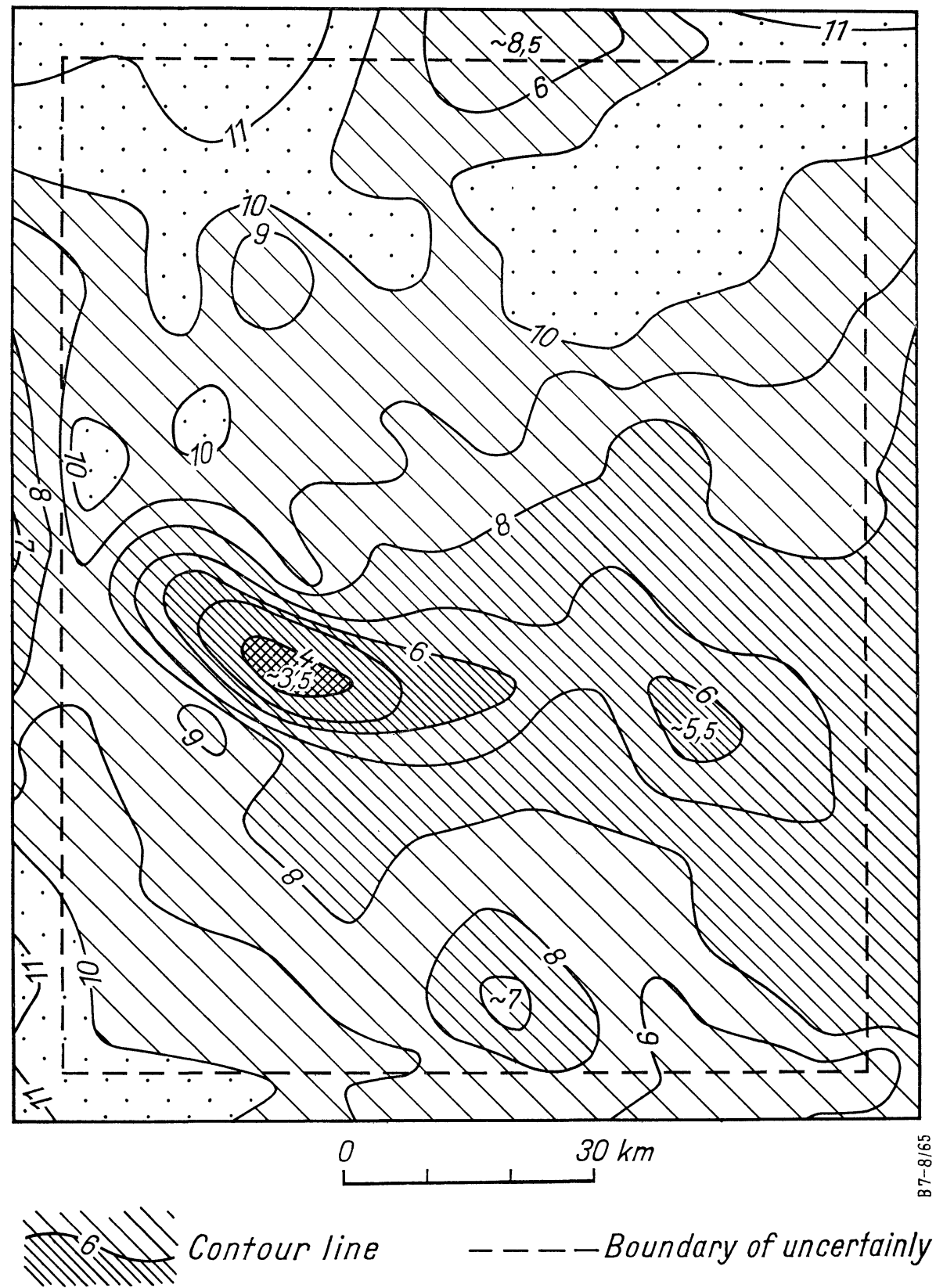

Fig. 8. Interpretation of the map Fig. 7. by a relief. Depth lines in $\mathrm{km}$ below sea level. Magnetization of the relief $J=130 \gamma$.

in Fig. 7. and 8. Fig. 7. represents the $\Delta T$-contour lines of an airborne measurement in $N W$ Germany. Two squares widely overlapping each other in the rectangular area were interpreted separately. The results were combined. Thereby it was possible to estimate the width of the stripe along the boundary where the results become uncertain. In Fig. 8. the combined results are shown together with the uncertainty stripe boundered by a dashed line. The 
starting depth for these reliefs had been chosen $\bar{z}=9 \mathrm{~km}$, the magnetization $J=130 \mathrm{r}$.

In most parts the relief seems to represent a surface within the crystalline rocks a few $\mathrm{km}$ higher than the Conrad-discontinuity which is found to lie at a depth of $11-15 \mathrm{~km}$ (Reich, 1950: Schulze \& Foertsch 1950: Dohr 1959) in areas where the present relief is at about $10 \mathrm{~km}$ depth. The high ascent of the relief to $3,5 \mathrm{~km}$ depth in the central part of the map is assumed to be a magnetized plutonic body possibly separated from the surrounding relief. Here the interpretation surface appears to be spread like a blanket over all magnetized bodies.

\section{3.-5. General remarks about the interpretation method}

For the choice of the starting depth there is no mathematical limit in downward direction because in the whole procedure only finite functions are involved. Now any field measurement containing measuring errors may be represented by a double sum of the type (5) within the interval of the measuring errors; hence it is impossible to give maximum depth estimates for any type of magnetic field measurements containing errors only from the shape of the field without further conditions relative to the magnetic sources.

Such conditions however can be assumed in agreement with geophysical or geological experience. One of the conditions is the limit of the magnetization values of the rocks, another is the improbability of the production of a simply-shaped anomaly by a complicated structure. These two conditions provide strong limitations for the starting depth values of the relief because with a given limit of the magnetization value the shape of the relief in too great a depht will show short-period oscillations with too high an amplitude as can be seen from equation (5). These oscillations, however can be made smaller than any given limit if the magnetization magnitude is chosen sufficiently big.

If, on the other hand, the starting depth has been chosen too small the relief will be too smooth. But a smooth relief is, of course, not impossible and it is evident that this method, like any other method, in general only provides a maximum depth value as far as no statistical considerations are made.

The computer time of one relief in a square containing $25 \times 25$ representative points is 5 minutes on an IBM 7090 computer. So it is possible to calculate several reliefs with different values $\bar{z}$ and $J$ and to choose the one which best fits the data obtained from other geophysical or geological investigations.

The main advantage of this method is that it is a direct one. So in cases where the presumptions of this method may be applicable the lenghty and tiring search for a valid model is replaced by a few 5 -minute-runs of a computer and the work of the geophysicist consists in judging several valid interpretations.

\section{A method for the interpretation of irregular fluctuations measured in geomagnetic profiles}

\section{4.-1. Geological and geophysical conditions in the area on hand of which the method has been developed.}

The irregular fluctuations treated here are of such type which for example is found in 
profiles measured over a large outcrop of rocks the magnetization of which is considerably inhomogeneous. Such conditions are found in the Vogelsberg-area in Germany. This area is built up by a sequence of basalt lava flows superposing each other. On the surface the basalt is covered by about $1-2 \mathrm{~m}$ of weathered basalt and Löss. In the fresh basalt we find troughs of some $100 \mathrm{~m}$ length and width and about $10-20 \mathrm{~m}$ depth filled with weathered basalt containing iron ore. The latter mainly occurs on former clefts of the basalt the structure of which is still visible although the material can be crushed between the fingers. The iron ore being less than $10 \%$ of the volume cannot be detected directly by any method of applied geophysics. But the search for ore is facilitated by the localization of those troughs. Now in its magnetization the fresh basalt shows much higher fluctuations than the weathered material and hence there was a difference in the fluctuations of the geomagnetic field in the area of outcropping fresh basalt on the one hand and in the area of the troughs on the other hand. The fluctuation character in the two different areas is demonstrated in Fig. 9. Two

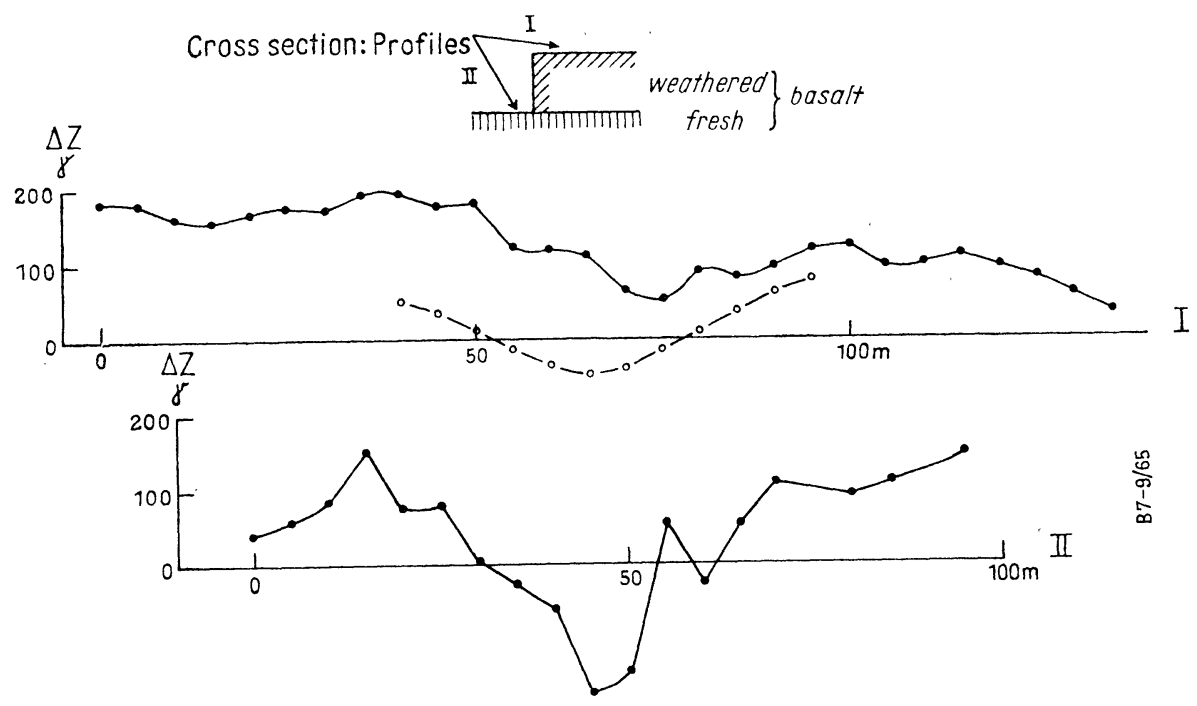

Fig. 9. $\Delta Z$-profiles in an open pit in the Vogelsberg on fresh basalt (II) and on weathered material (I). Dashed line: Continuation of profile II up to the level of profile I. (After Bentz, Lehrbuch der Angewandten Geologie, Bd. I. Stuttgart 1961)

profiles, measured in an open pit, are plotted. One of them right on top of the fresh basalt outcropping at the bottom shows high fluctuations. The other one, measured $8 \mathrm{~m}$ higher on weathered basalt, shows smaller fluctuations. The dashed line indicates a continuation of the lower profile up to the level of the upper profile. It shows smooth longperiodic fluctuations. From a comparison of the dashed line to the profile line measured on the weathered basalt it can be seen that the fluctuations in that profile are composed by the longperiodic ones originating from the deeplying fresh basalt, and the shortperiodic ones which are to come out of the weathered material. These latter fluctuations are smaller than those in the profile on the fresh basalt. This is a consequence of the weathering process which apparently has destroyed a big part of the magnetite of the fresh basalt. 
The inhomogeneity of the magnetization of the fresh basalt has been investigated by rock sample measurements. It was found that the biggest part of the inhomogeneity is due to the remanent magnetization. The magnitude of the difference vector of the remanent magnetization of two samples is dependent on the distance of the collecting locality. Of 150 pairs of samples with distances between 0 and $7 \mathrm{~m}$ preferably horizontal, the central values of groups lying in certain distance intervals were determined. A smooth curve through the central values passes at the distance $1 m$ the value $70 \gamma$, at $2 m 100 \gamma$, at $5 m 150 \gamma$. At greater distances the number of sample pairs is too small to produce statistically safe results. It can be expected, however, that the curve through the central values there approaches a final value of about $150-200 \mathrm{r}$ as far as the same lava flow is concerned.

In a vertical direction the magnitude of the remanent magnetization is ruled by the lava flows. Turkowsky has found that the magnitude of the remanent magnetization is decreasing from top to bottom in most cases in such a way that the upper half of the flow has about twice the magnitude of the lower half. The average thickness of the lava flow is about $10 \mathrm{~m}$. This, however is only a very rough description of Turkowsky's results which shall not be given in detail here. Angenheister has found that different lava flows are showing significant differences in the direction of the remanent magnetization.

From these observations the following picture of the inhomogeneity of the basalt can be designed: It is concluded that the basalt contains small volume elements with dimensions of some meters superposed by large volume elements with horizontal dimensions in the order of $100 \mathrm{~m}$ and a vertical extension of about $5 \mathrm{~m}$ which can be treated as homogeneously magnetized. The large parts are representing the upper and lower halfs of the lava flows; the small parts are standing for the inhomogeneity within the lava flows.

\section{4.-2. Definition of the average profile spectrum}

In the Vogelsberg area the vertical component of the earth's magnetic field $\Delta Z$ has been measured in ground profiles with torsion magnetometers Gfz (Askania-Werke, Berlin). The spacing between measuring points was $d=5 \mathrm{~m}$. The measuring points are counted by the integer $\rho$, so $\Delta Z(\rho)$ is the value measured in point number $\rho$.

Now for a quantitative treatment of the fluctuations of $\Delta Z(\rho)$ the profiles have been divided into parts of the length $r \cdot d=120 \mathrm{~m}$ each containing 25 points. These parts are counted by $p$. At first in each part the first and the last value are equalized by subtraction of a linear function:

$$
\Delta Z *(\rho)=\Delta Z(\rho)-\rho\left(\Delta Z\left(\rho_{p}+r\right)-\Delta Z\left(\rho_{p}\right)\right) / r
$$

$\rho_{p}$ being the number of the first point of part $p$. A harmonic analysis of the $r$ values $\Delta Z\left(\rho_{p}\right)$, $\ldots, \Delta Z\left(\rho_{p}+r-1\right)$ is then made which leads to the coefficients

$$
k_{n}=\frac{1}{r} \sum_{\rho=\rho p}^{\rho+r-1} \Delta Z *(\rho) \cdot \exp (-2 \pi \text { in } \rho / r)
$$

\footnotetext{
* In this older part of the paper a great deal of manual calculation had been involved. So for convenience the even number 24 had been chosen in contrary to the remark on page. 197.
} 
In this case we had chosen $r=24$; $^{*}$ the index $n$ is running from $-(r / 2-1)$ to $(r / 2-1)$ $k_{0}$ is the average of the $r$ values being of no interest here, the coefficient $k_{r} / 2$ must be treated in a special way and is not directly comparable to the other coefficients, so it shall be omitted.

The coefficients $k_{n}$ can be combined to real quantities because $k_{n}$ is the conjugate complex quantity of $k_{-n}$ :

$$
\begin{aligned}
& k_{n}+k_{-n}=2 \operatorname{Re}\left(k_{n}\right)=a_{n} \\
& k_{-n}-k_{n}=-2 \operatorname{Im}\left(k_{n}\right)=b_{n}
\end{aligned}
$$

Here $a_{n}$ and $b_{n}$ denote the wellknown real coefficients obtained by a harmonic analysis of the $r$ values $\Delta Z *(\rho)$. The profile spectrum of the part $p$ are the amplitudes

$$
c_{n}=\sqrt{a_{n}^{2}+b_{n}^{2}}=2 \sqrt{k_{n} \cdot k_{-n}} \quad n=1, \ldots, 11
$$

Now the average of any $c_{n}$ taken from all parts $p$ of profiles which are lying in a geologically homogeneous area (altogether $P$ parts) is calculated:

$$
\bar{c}_{n}=\sum_{p=1}^{P} c_{n}(p)
$$

The $\bar{c}_{n}$ form the average spectrum. Examples of profile spectra are plotted in Fig. 12.

\section{4.-3. Models for the average profile spectrum}

In order to obtain models for the measured profile spectra the structure of inhomogeneity of the magnetization distribution described at the end of para 4.1. is represented in a

\section{Jnterpretation of Profile Spectra}

Model for the distribution of magnetization built up by rectangular parallelepipeds with different magnetization

Characteristic data: Intervals for the dimensions of the parallelepipeds:

$$
\begin{array}{cc}
\text { Length in } x \text {-direction } & L_{1}<L<L_{2} \\
\text { Width in } y \text {-direction } & M_{1}<M<M_{2} \\
\text { Thickness in } z \text {-direction } & N
\end{array}
$$

Standard deviation of the

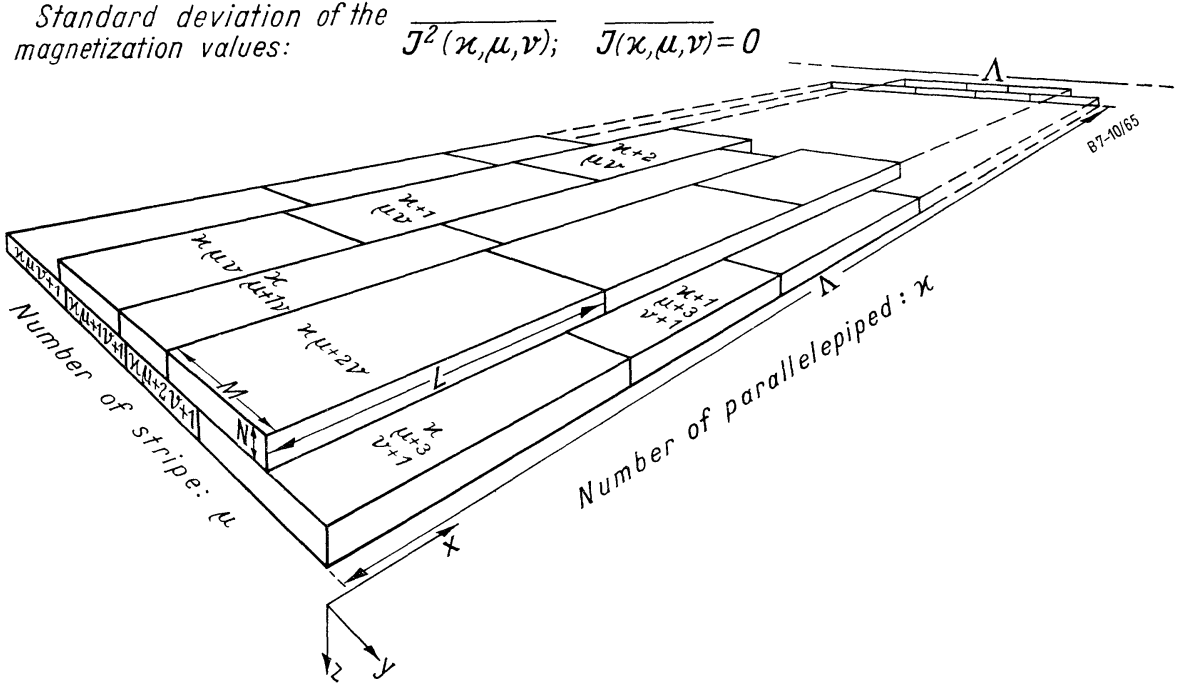

Fig. 10. Scheme of the model for an inhomogeneously magnetized thick layer. 
schematic way as it is shown in Fig. 10. The parts of homogeneous magnetization are rectangular parallelepipeds the horizontal dimensions of which are no fixed quantities but are uniformly distributed in intervals:

$$
\begin{array}{ll}
L_{1} \leqq L \leqq L_{2} & \text { (length parallel to } x \text {-axis) } \\
M_{1} \leqq M \leqq M_{2} & \text { (width parallel to } y \text {-axis) }
\end{array}
$$

The thickness $N$ parallel to the $z$-axis is a constant value. The parallelepipeds are counted by $\lambda$ in $x$-direction, by $\mu$ in $y$-direction, by $\nu$ in $z$-direction. $\nu=1$ denotes the uppermost layer of the parallelepipeds the upper boundary of which is the plane

$$
z=\bar{z} .
$$

The entire model is built up by $h$ layers;

$$
h N=T
$$

is the thickness of the model.

The $\lambda$ th part of the $\mu$ th row in the $\nu$ th layer has the magnetization $J(\lambda, \mu, \nu)$. The mean value of $J$ taken over a sufficiently big number of parts is zero, the standard deviation $\overline{J^{2}}$ is a characteristic quantity of the model. For the sake of simplicity the magnetization is taken vertical everywhere. Since in reality the directions of the magnetization of the lava flows are not strictly parallel and have an inclination of nearly $60^{\circ}$ (Angenheister, 1956) this simplification produces two systematic errors working one aginst the other:

1. If a variation of the direction would be supposed, besides the variation of the magnitude, the amplitude of the fluctuations would increase.

2. If the inclination would be supposed to be smaller than $90^{\circ}$ the magnetization waves striking North-South would produce smaller field waves than equal magnetization waves striking East-West. Hence the amplitude of the fluctuations would decrease.

Both effects are not very important. In the Vogelsberg the first one seems to be bigger, but probably not exceeding $15 \%$. That means: If the correct value of $\overline{J^{2}}$ is taken for a theoretical calculation the fluctuations will come out too small about $1 / 10$ because only the variations in magnitude but not the variations in direction are taken into accouut.

A further property of this model is its periodicity $\Lambda$ in $x$ and $y$. This renders possible a two-dimensional Fourier's analysis of the magnetization in horizontal layers and so an easy estimation of the expectancy of a two-dimensional field spectrum in a plane above the model. From this field spectrum the expectancy of a profile spectrum across this plane is then calculated. The field values in this profile again are periodical, but the real profiles show no periodical functions at all. Therefore from the spectrum of the periodical profile the spectrum of a quasinonperiodical part of this period is calculated. This, finally, can be compared to the measured spectra.

The expectance value of the two-dimensional amplitude spectrum $C_{m}{ }^{(k)}$ (see (9)) in a plane in the uppermost surface of the model $z=\bar{z}$ turns out to be

$$
E\left(C_{m}{ }^{(k) 2}\right)=E\left(4 K_{m}{ }^{(k)} \cdot K_{-m}{ }^{(-k)}\right)=J^{2} \frac{4}{\pi^{2} k^{2} m^{2}} \cdot \frac{4 A^{2}}{\left(L_{2}+L_{1}\right)\left(M_{2}+M_{1}\right)} \cdot
$$




$$
\begin{aligned}
& \left(1-\frac{\Lambda}{2 \pi k\left(L_{2}-L_{1}\right)}\left(\sin \left(2 \pi k L_{2} / \Lambda\right)-\sin \left(2 \pi k L_{1} / \Lambda\right)\right) .\right. \\
& \left(1-\frac{\Lambda}{2 \pi m\left(M_{2}-M_{1}\right)}\left(\sin \left(2 \pi m M_{2} / \Lambda\right)-\sin \left(2 \pi m M_{1} / \Lambda\right)\right) .\right. \\
& \frac{1-\exp \left(-2 \pi N \sqrt{k^{2}+m^{2}} / \Lambda\right)}{1+\exp \left(-2 \pi N \sqrt{k^{2}+m^{2}} / \Lambda\right)}\left(1-\exp \left(-4 \pi T \sqrt{k^{2}+m^{2}} / \Lambda\right)\right.
\end{aligned}
$$

(see Appendix 2, equ. (93)).

The transition from this two-dimensional spectrum to a spectrum of a profile running along the $x$-axis is done by the following procedure: From any field wave of (5)

$$
K_{m}{ }^{(k)} \exp (2 \pi i(k x+m y / \Lambda)
$$

(written for $z=z_{0}$ ) the $x$-axis will cut out the linear wave

$$
K_{k} \exp (2 \pi i k x / \Lambda) \text {. }
$$

All surface wave coefficients $K_{m}{ }^{(k)}$ with a fixed index $k_{1}$ are contributing towards this linear wave. All other waves of (5) with $k \neq k_{1}$ do not contribute because of the orthogonality. The phases of the wave coefficients $K_{m}{ }^{\left(k_{1}\right)}$ are independant of one another. If, now, in a great number of analysed squares the amplitudes $K_{m_{1}}{ }^{\left(k_{1}\right)}$ are distributed like the results of a twodimensional random walk, the resultant of all amplitudes with the index $k_{1}$ can be considered to be the result of a combination of random walks (Rayleigh, 1880) and has the expectancy value

$$
E\left(C_{m}{ }^{(k) 2}\right)=4 E\left(K_{k_{1}} \cdot K_{-k_{1}}\right)=4 \sum_{m=-R / 2}^{R / 2} K_{m}\left(k_{1}\right) \cdot K_{-m}{ }^{\left(-k_{1}\right)}
$$

Here $m$ is running from $-R / 2$ to $R / 2$ because the coefficients $K_{k}$ are considered to be determined from $R$ equidistant values, with the period $\Lambda$, given by

$$
d \cdot R=\Lambda(d \text { : spacing of the given values in the profile) }
$$

The $K_{k_{1}}$ form the spectrum of a profile measured along the $x$-axis. However, because of the regular arrangement of the parts in the model the $x$-axis is to be considered as a preferred direction. To avoid this disadvantage in equation (29) instead of the $K_{m}{ }^{\left(k_{1}\right)}$ average values are taken which are calculated as follows: From all amplitudes $K_{m}{ }^{(k)} \cdot K_{-m}{ }^{(-k)}$ with $n-0,5$ $<\sqrt{k^{2}+m^{2}} \leqq n+0,5$ the average value $V_{n}$ is calculated. Then the amplitudes $K_{m}{ }^{\left(k_{1}\right)} \cdot K_{-m}\left(-k_{1}\right)$ in (29) are replaced by $V_{n} \cdot\left(n+1-\sqrt{k^{2}+m^{2}}\right)+V_{n+1} \cdot\left(\sqrt{k^{2}+m^{2}}-n\right)$.

Inserting the squares of (31) in (30) the preference of a direction is cancelled:

$$
E\left(C^{2} k_{1}\right)=4 E\left(K_{k_{1}} \cdot K_{-k_{1}}\right)=4 \sum_{m=-R / 2}^{R / 2}\left(V_{n} \cdot\left(\sqrt{k^{2}+m^{2}}-n-1\right)+V_{n+1} \cdot\left(\sqrt{k^{2}+m^{2}}-n\right)\right)^{2}
$$

This is the expectancy of the spectrum of a profile measured over a model with the period $A$ in $x$ and $y$ but without a preferred direction in its structure. This profile also shows the period $\Lambda$. But a part of the profile, essentially smaller than $\Lambda$ may be considered as a part of a nonperiodic function. In the following the part $\Lambda / 3$ has been adopted which at both ends has intervals of the same length with generally completely different values. 
So for comparison to the nonperiodic measured profiles the amplitudes $k_{n}$ in the short interval $\lambda=\Lambda / 3$ are calculated starting from the values (32). The function from which a part shall be analysed has the form

$$
\sum_{N=-R / 2}^{R / 2} K_{N} \exp (2 \pi i N \rho / R)
$$

The interval of analysis has the length

$$
\lambda=\Lambda / 3=d \cdot R / 3=d \cdot r ; \quad r=R / 3
$$

According to the definition of the average profile spectrum (23)-(27) the values at both ends of the analyzed interval must be equalized by subtraction of a linear function:

$$
\Delta Z *(\rho)=\sum_{N=-R / 2}^{R / 2} K_{N}\left(\exp (2 \pi i N \rho / 3 r)-\frac{\rho}{r}(\exp (2 \pi i N / 3)-1)\right)
$$

The analysis of this function according to (24) yields the coefficients

$$
\begin{gathered}
k_{n}=\frac{1}{r} \sum_{\rho=0}^{r-1} \sum_{N=-R / 2}^{R / 2} \exp (-2 \pi i n \rho / r) \cdot \\
K_{N}\left(\exp (2 \pi i N \rho / 3 r)-\frac{\rho}{r}(\exp (2 \pi i N / 3)-1)\right)
\end{gathered}
$$

By exchange of these two summations and evaluation of the sum over $\rho k_{n}$ is obtained as a weighed sum of the $K_{N}$. The transition to the real amplitudes $c_{n}$ is done by multiplying $k_{n}$ by $k_{-n}$ (see (26)). In the expectancy of this product only the terms with the products $K_{N} \cdot K_{-N}$ are unequal zero. By inserting the values (32) in (36) the expectancy of the amplitudes $c_{n}$ turns out to be

$$
\begin{aligned}
E\left(c_{n}{ }^{2}\right) & =4 E\left(k_{n} k_{-n}\right)=4 E\left(K_{3 n} K_{-3 n}\right) \\
& +4 \sum_{\substack{N=-R / 2 \\
N \neq 3 n}}^{R / 2} E\left(K_{N} K_{-N}\right)\left[\frac{\sin (\pi N / 3) \cdot \sin (\pi N / 3 r)}{\sin (\pi n / r)}\right]^{2} \cdot\left[\frac{1}{\sin (\pi(N-3 n) / 3 r)}\right]^{2}
\end{aligned}
$$

This result is deduced from (36) in Appendix 3. It represents the expectancy of a profile spectrum measured above a model of the structure described at the beginning of para 4.3, p.208/9, with a quasi-nonperiodic function in the profile. This spectrum is obtained in the same way as the spectra of the measured profiles and may be compared to them.

Fig. 11 shows three sets of profile spectra calculated in this way for different models characterized by the dimensions of the volume parts of homogeneous magnetization. The parameter of these sets is the elevation of the profiles above the uppermost layer of the model. At the spectra in small elevations it can be seen that above the models with large volume parts the long waves are more pronounced whereas above the models with the small parts the short waves show higher amplitudes. The spacing of the amplitudes dependent on the elevation is smaller for long waves than for short ones; the spacing of the spectra is over the large volume parts smaller than over the small ones. So it is evident that two profiles measured on the same track but in different elevations will give more information about the structure of the underground than only one can do. 


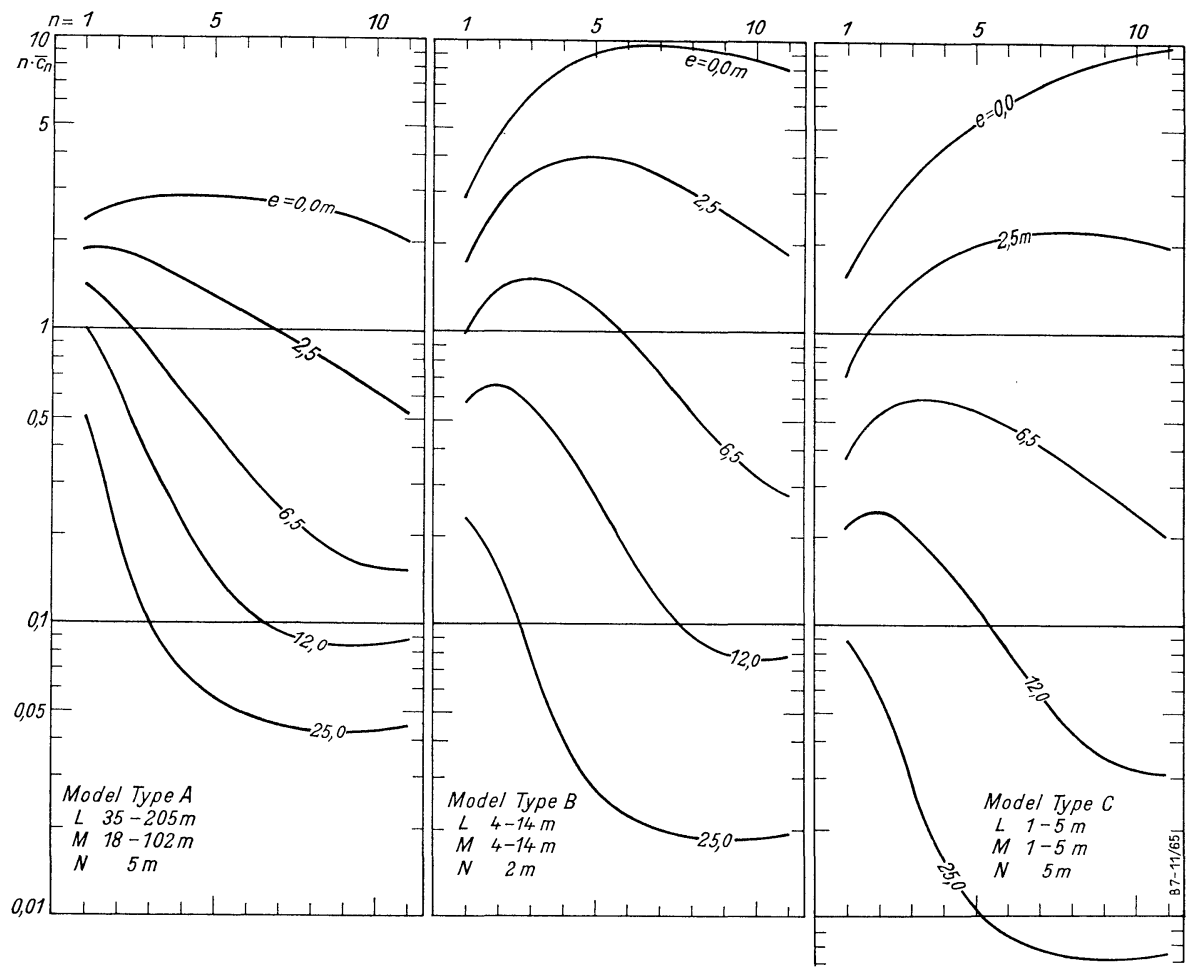

Fig. 11. Three sets of theoretical profile spectra calculated for different model types as indicated. Parameter of the sets : $e=$ elevation of the profile above the model.

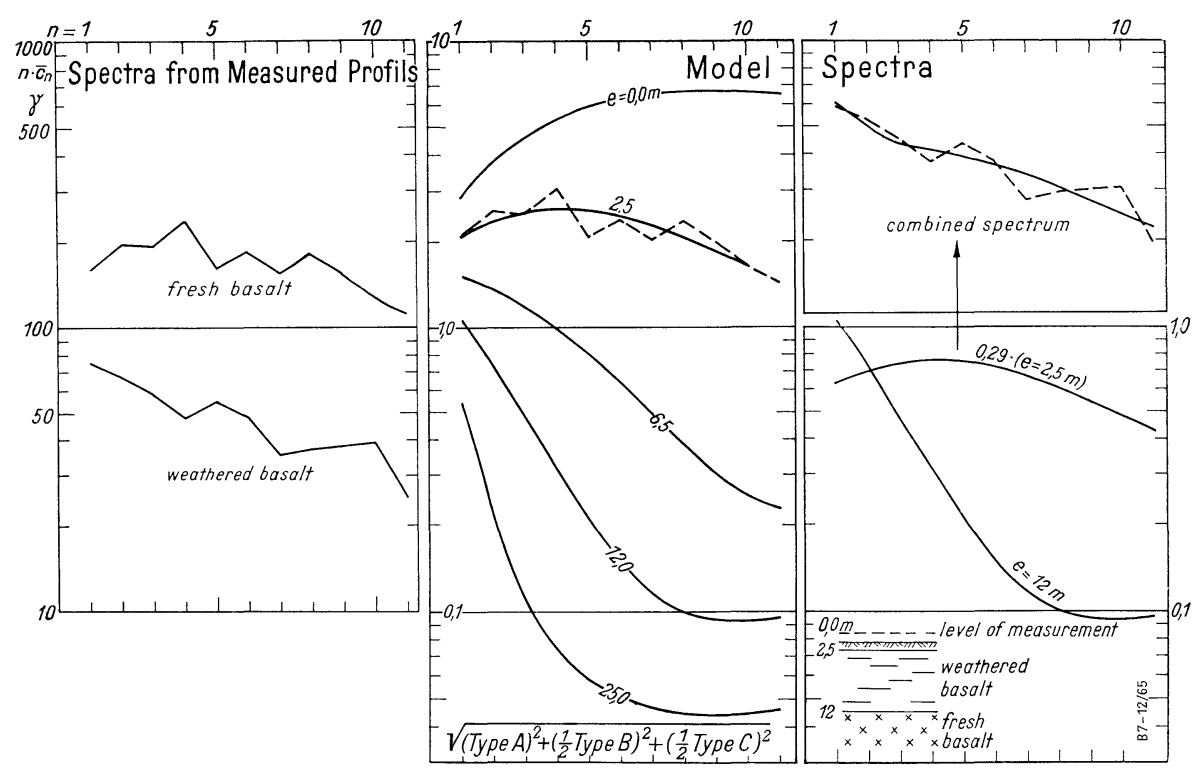

Fig. 12. Interpretation of two spectra from measured profiles (left). The upper spectrum is compared to a theoretical spectrum $2,5 \mathrm{~m}$ above a model combined from the three types $A, B$ and $C$ of Fig. 11. (middle). The lower spectrum is interpreted as a combination of two spectra in the elevations $e=2,5 \mathrm{~m}$ and $e=12 \mathrm{~m}$ above the model (right). At the bottom a geological profile of interpretaion is plotted. 


\section{4.-4. Application to measured profiles}

The application of these model spectra of the profiles measured in the Vogelsberg is shown in Fig. 12. From results of drillholes and small exploration shafts is to be estimated that the magnetized material is to be expected in a depth of 1 to $1,5 \mathrm{~m}$. Together with the height of the measuring instrument of about $11 / 4 m$ this means an elevation of $2,5 \mathrm{~m}$ of the profile above the model. Now the profile spectrum measured over fresh basalt cannot be compared in any of the calculated sets to a spectrum in that elevation. But this was not to be expected because from the studies of rock samples it was concluded that small and large volume parts should contribute to the inhomogeneity of the magnetization.

Hence a combination of the three sets was calculated whereby the different spectra were weighed as indicated, the squares of these values were added and the square root of the sum was taken. With the weighing factors $1,0,5 ; 0,5$ for the model types $A, B, C$, respectively, a fairly good agreement was found.

For the interpretation of the profile spectra measured over troughs with weathered basalt two model spectra must be combined according to the remarks made for Fig. 9. So for the representation of the deeplying fresh basalt the model spectrum at elevation $12 \mathrm{~m}$ has been taken whereas for the weathered material the spectrum at elevation $2,5 \mathrm{~m}$ with the weighing factor 0,29 has been chosen. The combined spectrum shown also in Fig. 12 is in sufficiently good agreement with the measured spectrum.

From these two comparisons a value for the standard deviation of the magnetization values of the model's volume parts could be obtainded which in the first case (profile over fresh basalt) is $\sqrt{\overline{J^{2}}}=78 \gamma$, in the second case (profile over trough) $\sqrt{\overline{J^{2}}}=61 \gamma$ (deviation of the model combined by the types $A, B, C$ ). The second value is considerably smaller than the first one. This discrepancy can be caused by a systematic difference of the basalt occurring in the two neighbouring areas. But it could also be produced by the assumption of too small a depth for the surface of the fresh basalt beneath the trough. A better agreement would be reached with a depth assumption of $15-20 \mathrm{~m}$. In this case, however, the shape of the combined spectrum curve (Fig. 12, right) would show a greater difference to the measured spectrum.

\section{4.-5. Comparison to rock sample measurements}

The standard deviation obtained from the profile over fresh basalt can be compared to the results of the rock sample measurements. For this purpose the following distribution function for the magnetization values of the volume parts is adopted:

$$
h(J)=4 \alpha \sqrt{\frac{\alpha}{\pi}} J^{2} \exp \left(-\alpha J^{2}\right)
$$

The mean value of this distribution is

$$
\bar{J}=\frac{2}{\sqrt{\alpha \pi}},
$$

the standard deviation is 


$$
\overline{J^{2}}=\frac{1}{\alpha}(3 / 2-4 / \pi)
$$

Inserting the value $\sqrt{\overline{J^{2}}}=78 \gamma$ in (39) the mean value of $J$ is found to be

$$
\bar{J}=225 \gamma \text {. }
$$

This value is expected to be too big for about $10-15 \%$ because the direction of magnetization of all model parts has been assumed to be vertical (see p. 209). The corrected value is $\bar{J} \approx 200 \gamma$. This is in fairly well agreement with Angenheister's results (1956). He found in this area (westlich der Seentallinie) for the remanent magnetization the mean value $\overline{J_{r}}=$ $218 \gamma$. For the induced magnetization he found $\overline{J_{i}}=43 \gamma$. The induced magnetization apparently contributes to the inhomogeneity less than the remanent magnetization, so the mean value of magnetization comparable to the result obtained from the model can be bigger than $218 r$ only by a small amount.

The weighing factor 0,29 (see Fig. 12) representing the relation of the magnetization of the weathered material to that of the fresh basalt from rock sample measurements must be expected to be $0,2-0,35$ (Angenheister, 1957).

The results given here show a surprisingly good agreement. This must be taken as incidental because the real distribution of magnetization could be represented by the model only in a rather rough way. If only the results to be compared would have had an equal order of magnitude we might have been satisfied already.

\section{4.-6. Conclusions about the method}

Irregular fluctuations of the earth's magnetic field observed in profile measurements can be described by the profile spectrum (defined by equ. (23) - (27)) and interpreted by comparison to general three-dimensional models of an inhomogeneously magnetized thick layer. The profile spectra can be continued upward and downward if the general distribution of the magnetization is known. On the other hand the ambiguity of the interpretation is reduced if profiles measured in different elevations are available for the same rock type.

\section{Acknowledgement}

The author is very much indebted to Messrs. Dr. Bosum, Kind and Dr. Mundry for helpful discussions and for carrying out the necessary computer calculation.

\section{Appendix 1}

The field wave produced by a magnetization wave in a half space

For the following consideration of one partial field wave with the indices $k, m$ an orthogonal coordinate system $s, t, u$ is adopted: The $s$-axis is horizontal; the angle $\alpha+\delta$ (see (11)) between the $s$-axis and the magnetic meridian is positive if the $s$-axis lies East of the meridian. The $u$-axis is pointing downward. The $t$-axis is perpendicular to $s$ and $u$. The vector of the earth's magnetic field in this system is described by 


$$
\begin{array}{ll}
s \text {-component: } & T \cos I \cos (\alpha+\delta) \\
t \text {-component: } & T \cos I \sin (\alpha+\delta) \\
u \text {-component: } & T \sin I
\end{array}
$$

$I$ is the inclination of the earth's magnetic field. The projection of the field to the $s, u$ plane has the inclination $I^{\prime}$. We write three relations of $I^{\prime}$ and $I$ :

$$
\begin{aligned}
& \cos I^{\prime}=\cos I \cos (\alpha+\delta) / \sqrt{\sin ^{2} I+\cos ^{2} I \cos ^{2}(\alpha+\delta)} \\
& \sin I^{\prime}=\sin I / \sqrt{\sin ^{2} I+\cos ^{2} I \cos ^{2}(\alpha+\delta)} \\
& \cot I^{\prime}=\cot I \cos (\alpha+\delta)
\end{aligned}
$$

The coordinate system is chosen in such a way that the field wave under consideration is depenent on the s-coordinate, only. Therefore, instead of the indices $k, m$ the abbreviation $n=\sqrt{k^{2}+m^{2}}$ is taken as index and frequency number. With this quantity the partial field wave (8) can be rewritten as follows

$$
P_{n} \cos (2 \pi n s / \Lambda)+Q_{n} \sin (2 \pi n s / \Lambda)
$$

Now according to Nagata (1938) this field wave in the plane $u=\bar{u}$ can be interpreted by a magnetization wave in the half space $u>\bar{u}$ which shall be described by the components of magnetization :

$$
\begin{array}{ll}
s \text {-component: } & J^{(s)}=J_{n}{ }^{(s)} \cos \left(2 \pi n s / \Lambda-\varphi_{n}{ }^{(s)}\right) \\
t \text {-component: } & J^{(t)}=J_{n}{ }^{(t)} \cos \left(2 \pi n s / \Lambda-\varphi_{n}{ }^{(t)}\right) \\
u \text {-component: } & J^{(u)}=J_{n}{ }^{(u)} \cos \left(2 \pi n s / \Lambda-\varphi_{n}{ }^{(u)}\right)
\end{array}
$$

These magnetization distributions are dependent on variations of $s$ only and have the same direction and wavelength as the field wave (43). The magnetization is assumed to be parallel to the earth's magnetic field everywhere. Consequently all waves (44) have the same phase $\varphi_{n}$. Moreover the amplitudes of the magnetization components are proportional to those of the field (41):

$$
\begin{aligned}
& J_{n}{ }^{(s)}=J_{n} \sin (\pi / 2-I) \cos (\alpha+\delta) \\
& J_{n}{ }^{(t)}=J_{n} \sin (\pi / 2-I) \sin (\alpha+\delta) \\
& J_{n}{ }^{(u)}=J_{n} \cos (\pi / 2-I)
\end{aligned}
$$

The $t$-component running parallelly to the boundary plane $u=\bar{u}$ and parallelly to the vertical planes of constant magnetization cannot produce a field in the space $u \leqq \bar{u}$. Hence the effective components are the $s$-and the $u$-component. The amplitudes and the phases of these components are combined now to complex quantities:

$$
\begin{aligned}
& S_{n}=1 / 2 J_{n}{ }^{(s)} \exp \left(i \varphi_{n}\right) \\
& U_{n}=1 / 2 J_{n}{ }^{(u)} \exp \left(i \varphi_{n}\right)
\end{aligned}
$$

The factor $1 / 2$ is introduced in analogy to (9).

The quotient $S_{n} / U_{n}$ turns out to be (see (45) and (42))

$$
S_{n} / U_{n}=J_{n}{ }^{(s)} / J_{n}{ }^{(u)}=\tan (\pi / 2-I) \cos (\alpha+\delta)=\tan \left(\pi / 2-I^{\prime}\right)
$$

Now the complex Amplitudes $S_{n}$ and $U_{n}$ are again combined to one complex quantity: 


$$
M_{n}=U_{n}-i S_{n}
$$

Regarding (46) and (47) this can be rewritten

$$
\begin{aligned}
M_{n} & =\sqrt{{U_{n}{ }^{2}+S_{n}^{2}}^{2}} \exp \left(-i\left(\pi / 2-I^{\prime}\right)\right)=1 / 2 \sqrt{J_{n}{ }^{(s) 2}+J_{n}{ }^{(u) 2}} \exp \left(i\left(\varphi_{n}-\pi / 2+I^{\prime}\right)\right) \\
& =1 / 2 J_{n} \sqrt{\sin ^{2} I+\cos ^{2} I \cos ^{2}(\alpha+\delta)} \exp \left(i\left(\varphi_{n}-\pi / 2+I^{\prime}\right)\right) \\
& =\left|M_{n}\right| \exp \left(i\left(\varphi_{n}-\pi / 2+I^{\prime}\right)\right)
\end{aligned}
$$

where $\left|M_{n}\right|$ denotes the magnitude of $M_{n}$ :

$$
\left|M_{n}\right|=1 / 2 J_{n} \sqrt{\sin ^{2} I+\cos ^{2} I \cos ^{2}(\alpha+\delta)} .
$$

Now the relation of the anomalous vector $\delta T$ lying in the $s, u$-plane and the corresponding magnetization wave (44) can be formulated quite simply

$$
\delta T=4 \pi M_{n} \exp (-i 2 \pi n s / \Lambda)=4 \pi\left|M_{n}\right| \exp \left(-i\left(2 \pi n s / \Lambda-\varphi_{n}+\pi / 2-I^{\prime}\right)\right)
$$

The real component of (51) is lying parallel to the $u$-axis, the imaginary one parallel to the $s$-axis. The projection of $\delta T$ to the direction of the earth's magnetic field projected to the $s, u$-plane is carried out by multiplying $\delta T$ by the conjugate complex quantity of the unity vector in this direction and taking the real part of the product:

$$
\Delta T^{(s)}=\operatorname{Re}\left(\delta T \exp \left(-\mathrm{i}\left(\pi / 2-I^{\prime}\right)\right)=4 \pi\left|M_{n}\right| \cos \left(2 \pi n s / \Lambda-\varphi_{n}+\pi-2 I^{\prime}\right)\right.
$$

The projection of $\Delta T^{(s)}$ to the direction of the earth's magnetic field is done by multiplying $\Delta T^{(s)}$ by the factor $\sqrt{\sin ^{2} I+\cos ^{2} I \cos ^{2}(\alpha+\delta)}$ which is the same factor as has been used in the projection of the entire magnetization amplitude $J_{n}$ to the $s, u$-plane (see (49)) :

$$
\begin{aligned}
\Delta T & =\Delta T^{(s)} \sqrt{\sin ^{2} I+\cos ^{2} I \cos ^{2}(\alpha+\delta)}, \text { or, by inserting (50) in (52) } \\
& =2 \pi J_{n}\left(\sin ^{2} I+\cos ^{2} I \cos ^{2}(\alpha+\delta)\right) \cos \left(2 \pi n s / \Lambda-\varphi_{n}+\pi-2 I^{\prime}\right)
\end{aligned}
$$

For a calculation of the anomaly of the vertical component $\Delta Z$ only the real part of (51) must be taken:

or, regarding (50)

$$
\Delta Z=4 \pi\left|M_{n}\right| \cos \left(2 \pi n s / \Lambda-\varphi_{n}+\pi / 2-I^{\prime}\right)
$$

$$
\Delta Z=2 \pi J_{n} \sqrt{\sin ^{2} I+\cos ^{2} I \cos ^{2}(\alpha+\delta)} \cos \left(2 \pi n s / \Lambda-\varphi_{n}+\pi / 2-I^{\prime}\right)
$$

As can be seen from (53) and (54) the phase of the field wave is shifted with respect to the magnetization wave and, in the case of $\Delta T$, by $\pi-2 I^{\prime}$, in the case of $\Delta Z$ by $\pi / 2-I^{\prime}$. The maximum of the field wave is lying South of the corresponding maximum of the magnetization wave in areas where $45^{\circ}<I \leqq 90^{\circ}$.

It shall be repeated that the foregoing procedure is no deduction of the relation between field and magnetization. The equations (51) - (54) are nothing but a very short formulation of Nagata's results (1938) using the symbols of the present paper.

For the purpose of establishing a computer program the phase $\varphi_{m}{ }^{(k)}$ and the phase shift in (53) and (54) should be rewritten in coefficients of the sin-and the cos-component of the field wave $P_{m}{ }^{(k)}, Q_{m}{ }^{(k)}$ and of the magnetization wave $J_{m}{ }^{(k)} \sin \varphi_{m}{ }^{(k)}, J_{m}{ }^{(k)} \cos \varphi_{m}{ }^{(k)}$. These expressions should involve besides the indices $k, m$ only quantities which do not depend on $k, m$. 
By developing the last cos-function of (53) and writing it as a function of $x$ and $y$ with the indices $k$ and $m$ we find

$$
\begin{aligned}
& \cos \left(2 \pi(k x+m y) / \Lambda-\varphi_{m}{ }^{(k)}+\pi-2 I^{\prime}\right)=\cos \varphi_{m}{ }^{(k)}\left[\cos \left(\pi-2 I^{\prime}\right) \cos (2 \pi(k x+m y) / \Lambda)\right. \\
& \left.\quad-\sin \left(\pi-2 I^{\prime}\right) \sin (2 \pi(k x+m y) / \Lambda)\right]+\sin \varphi_{m}{ }^{(k)}\left[\sin \left(\pi-2 I^{\prime}\right) \cos (2 \pi(k x+m y) / \Lambda)\right. \\
& \left.\quad+\cos \left(\pi-2 I^{\prime}\right) \sin (2 \pi(k x+m y) / \Lambda)\right] .
\end{aligned}
$$

Regarding the relations (42) the quantities $\sin \left(\pi-2 I^{\prime}\right)$ and $\cos \left(\pi-2 I^{\prime}\right)$ can be formulated in terms of $I$ and $\alpha+\delta$ :

$$
\begin{aligned}
& \sin \left(2\left(\pi / 2-I^{\prime}\right)\right)=2 \sin \left(\pi / 2-I^{\prime}\right) \cos \left(\pi / 2-I^{\prime}\right) \\
& \quad=2 \sin I \cos I \cos (\alpha+\delta) /\left(\sin ^{2} I+\cos ^{2} I \cos ^{2}(\alpha+\delta)\right) \\
& \cos \left(2\left(\pi / 2-I^{\prime}\right)=\cos ^{2}\left(\pi / 2-I^{\prime}\right)-\sin ^{2}\left(\pi / 2-I^{\prime}\right)\right. \\
& \quad=\left[\sin ^{2} I-\cos ^{2} I \cos ^{2}(\alpha+\delta)\right] /\left(\sin ^{2} I+\cos ^{2} I \cos ^{2}(\alpha+\delta)\right)
\end{aligned}
$$

Inserting (56) in (55) and this again in (53) leads to

$$
\begin{aligned}
& \Delta T=2 \pi J_{m}{ }^{(k)}\left\{\operatorname { c o s } \varphi _ { m } { } ^ { ( k ) } \left[\left(\sin ^{2} I-\cos ^{2} I \cos ^{2}(\alpha+\delta)\right) \cos (2 \pi(k x+m y) / \Lambda)\right.\right. \\
& \quad-2 \sin I \cos I \cos (\alpha+\delta) \sin (2 \pi(k x+m y) / \Lambda)] \\
& \quad+\sin \varphi_{m}{ }^{(k)}[2 \sin I \cos I \cos (\alpha+\delta) \cos (2 \pi(k x+m y) / \Lambda) \\
& \left.\quad+\left(\sin ^{2} I-\cos ^{2} I \cos ^{2}(\alpha+\delta)\right) \sin (2 \pi(k x+m y / \Lambda)]\right\}
\end{aligned}
$$

In the case of $\Delta Z$ instead of (55) we yield

$$
\begin{aligned}
& \cos \left(2 \pi(k x+m y) / \Lambda-\varphi_{m}{ }^{(k)}+\pi / 2-I^{\prime}\right) \\
& \quad=\cos \varphi_{m}{ }^{(k)}\left[\cos \left(\pi / 2-I^{\prime}\right) \cos (2 \pi(k x+m y) / \Lambda)\right. \\
& \left.\quad-\sin \left(\pi / 2-I^{\prime}\right) \sin (2 \pi(k x+m y) / \Lambda)\right] \\
& \quad+\sin \varphi_{m}{ }^{(k)}\left[\sin \left(\pi / 2-I^{\prime}\right) \cos (2 \pi(k x+m y) / \Lambda)\right. \\
& \left.\quad+\cos \left(\pi / 2-I^{\prime}\right) \sin (2 \pi(k x+m y) / \Lambda)\right]
\end{aligned}
$$

and with the relations (42) we find:

$$
\begin{aligned}
& \Delta Z=2 \pi J_{m}{ }^{(k)}\left\{\cos \varphi_{m}{ }^{(k)}[(\sin I \cos (2 \pi(k x+m y) / \Lambda)\right. \\
& \quad-\cos I \cos (\alpha+\delta) \sin (2 \pi(k x+m y) / \Lambda)] \\
& \quad+\sin \varphi_{m}{ }^{(k)}[(\cos I \cos (\alpha+\delta) \cos (2 \pi(k x+m y) / \Lambda) \\
& \quad+\sin I \sin (2 \pi(k x+m y) / \Lambda)]\} \\
& \sqrt{\sin ^{2} I+\cos ^{2} I \cos ^{2}(\alpha+\delta) .}
\end{aligned}
$$

In (57) and (59) $\cos (\alpha+\delta)$ is to be expressed in terms of $k, m$ and $\delta$ :

$$
\cos (\alpha+\delta)=\frac{k \cos \delta-m \sin \delta}{\sqrt{k^{2}+m^{2}}}
$$

In practical interpretation work we start with a representation of the measured field component in the form (8)

$$
\Delta T=P_{m}{ }^{(k)} \cos (2 \pi(k x+m y) / \Lambda)+Q_{m}{ }^{(k)} \sin (2 \pi(k x+m y) / \Lambda) .
$$

Writing this expression on the left side of (57) we obtain by comparison of coefficients 


$$
\begin{aligned}
& P_{n}{ }^{(k)}=2 J_{m}{ }^{(k)}\left\{\cos \varphi_{m}{ }^{(k)}\left[\sin ^{2} I-\cos ^{2} I \cos ^{2}(\alpha+\delta)\right]\right. \\
& \left.\quad+\sin \varphi_{m}{ }^{(k)} \cdot 2 \sin I \cos I \cos (\alpha+\delta)\right\} \\
& Q_{m}{ }^{(k)}=2 J_{m}{ }^{(k)}\left\{\cos \varphi_{m}{ }^{(k)}[-2 \sin I \cos I \cos (\alpha+\delta)]\right. \\
& \left.\quad+\sin \varphi_{m}{ }^{(k)}\left[\sin ^{2} I-\cos ^{2} I \cos ^{2}(\alpha+\delta)\right]\right\}
\end{aligned}
$$

In the case of $\Delta Z=P_{m}{ }^{(k)} \cos (2 \pi(k x+m y) / \Lambda)+Q_{m}{ }^{(k)} \sin (2 \pi(k x+m y) / \Lambda)$ it is found

$$
\begin{aligned}
P_{m}{ }^{(k)} & =\frac{2 J_{m}{ }^{(k)}\left[\cos \varphi_{m}{ }^{(k)} \sin I+\sin \varphi_{m}{ }^{(k)} \cos I \cos (\alpha+\delta)\right]}{\sqrt{\sin ^{2} I+\cos ^{2} I \cos ^{2}(\alpha+\delta)}} \\
Q_{m}{ }^{(k)} & =\frac{\left.2 J_{m}{ }^{(k)}\left[\cos \varphi_{m}{ }^{(k)}(-\cos I \cos (\alpha+\delta))+\sin \varphi_{m}{ }^{(k)} \sin I\right)\right]}{\sqrt{\sin ^{2} I+\cos ^{2} I \cos ^{2}(\alpha+\delta)}}
\end{aligned}
$$

In order to obtain the coefficients of the magnetization wave $J_{m}{ }^{(k)} \cos \varphi_{m}{ }^{(k)}$ and $J_{m}{ }^{(k)}$ $\sin \varphi_{m}{ }^{(k)}$ the equation systems (61) or (62) are resolved with respect to these quantities, according to the rules of the elementary algebra. Inserting (60) in these solutions we will find expressions for the coefficients of the magnetization waves involving only the results of the analysis (6) and (9): $P_{m}{ }^{(k)}, Q_{m}{ }^{(k)}$, the indices $k, m$ and the quantities $\sin I, \cos I, \sin \delta, \cos \delta$ which do not depend on $k, m$. These expressions are looking rather complicated but they are best suited for a computer program.

\section{Appendix 2}

Calculation of the expectancy value of the amplitude spectrum $K_{m}{ }^{(k)} \cdot K_{-m}{ }^{(-k)}$ of the magnetic field above the model shown in Fig. 9.

The first step of this calculation is a two-dimensional Fourier's analysis of the magnetization distribution of a thin horizontal plate lying within the model. From the resulting magnetization waves the contribution to the corresponding field waves in a plane above the model is calculated as the next step. The integral over the entire vertical extent of the model leads to the required spectrum for which the expectany is calculated as the last step.

\section{A. Fourier's analysis of the magnetization in the level $z$}

The coefficients of this analysis shall be defined in analogy to (6) by

$$
J_{m}{ }^{(k)}(z)=\frac{1}{\Lambda^{2}} \int_{0}^{\Lambda} \int_{0}^{\Lambda} \exp (-2 \pi i(k x+m y) / \Lambda) \cdot J(x, y, z) d x \cdot d y
$$

whereby $A$ denotes the period of the model in $x$ - and $y$ - direction, $k$ and $m$ are integers running from $-\infty$ to $+\infty$. Now the model is divided into volume parts with homogeneous magnetization, hence the inner integral of (63) can be replaced by a sum:

$$
J_{m}{ }^{(k)}(z)=\frac{1}{\Lambda^{2}} \int_{0}^{\Lambda} \sum_{\lambda=1}^{l(\mu \nu)} J(\lambda, y, z) \int_{x_{\lambda-1} \mu_{\nu}}^{x_{\lambda \mu_{\nu}}} \exp (-2 \pi i(k x+m y) / \Lambda) d x \cdot d y
$$

Here $l(\mu, \nu)$ denotes the number of volume parts within one period $A$ in the level $z$ lying within the $\nu$ th layer of volume parts and containing the straight line $y=$ const in the $\mu$ th row of parts. $J(\lambda, y, z)$ are the magnitudes of magnetization of these parts, $x_{\lambda^{\mu} \nu}$ is the $x$ - 
coordinate at the boundary of the $\lambda$ th and the $(\lambda+1)$ th part, $x_{o \mu_{\nu}}=0$.

By splitting the exponential function in (64) into its parts dependent on $x$ and $y$ and evaluating the integral over $x$ we yield

$$
\begin{aligned}
& J_{m}{ }^{(k)}(z)=\frac{1}{\Lambda^{2}} \int_{0}^{\Lambda} \exp (-2 \pi i \cdot m y / \Lambda) \sum_{\lambda=1}^{l(\mu \nu)} J(\lambda, y, z) \cdot \\
& \frac{i \Lambda}{2 \pi k}\left(\exp \left(-2 \pi i \cdot k x_{\lambda \mu_{\nu}} / \Lambda\right)-\exp \left(-2 \pi i \cdot k x_{\lambda-1 \mu_{\nu}} / \Lambda\right)\right) \mathrm{dy} .
\end{aligned}
$$

The $y$-integral is evaluated in a similar way leading to

$$
\begin{aligned}
J_{m}{ }^{(k)}(z) & =\frac{1}{\Lambda^{2}} \sum_{\mu=1}^{\mathrm{g}(\nu)} \sum_{\lambda=1}^{l(\mu, \nu)} J(\lambda, \mu, z) \frac{-\Lambda^{2}}{4 \pi^{2} k m} \cdot \\
& \left(\exp \left(-2 \pi i \cdot k x_{\lambda^{\mu \nu}} / \Lambda\right)-\exp \left(-2 \pi i \cdot k x_{\lambda-1 \mu_{\nu}} / \Lambda\right)\right) \cdot \\
& \left(\exp \left(-2 \pi i \cdot m y_{\mu_{\nu}} / \Lambda\right)-\exp \left(-2 \pi i \cdot m y_{\mu_{-1 \nu}} / \Lambda\right)\right)
\end{aligned}
$$

The number $g(\nu)$ is the number of the rows of volume parts counted along the $y$-axis (see Fig. 9), $y_{\mu_{\nu}}$ are the $y$-coordinates at the boundaries of these rows. With the abbreviations

$$
\begin{aligned}
& D_{1}\left(x_{\lambda \mu_{\nu}}\right)=\exp \left(-2 \pi i \cdot k x_{\lambda \mu_{\nu}} / \Lambda\right)-\exp \left(-2 \pi i \cdot k x_{\lambda-1 \mu_{\nu}} / \Lambda\right) \\
& D_{2}\left(y_{\mu_{\nu}}\right)=\exp \left(-2 \pi i \cdot m y_{\mu_{\nu}} / \Lambda\right)-\exp \left(-2 \pi i \cdot m y_{\mu_{-1 \nu}} / \Lambda\right)
\end{aligned}
$$

(66) can be rewritten to

$$
J_{m}{ }^{(k)}(z)=\frac{-1}{4 \pi^{2} k m} \sum_{\mu=1}^{g(\nu)} \sum_{\lambda=1}^{l(\mu, \nu)} J(\lambda, \mu, z) \cdot D_{1}\left(x_{\lambda \mu_{\nu}}\right) \cdot D_{2}\left(y_{\mu_{\nu}}\right)
$$

\section{B. The field wave corresponding to (67) in the level $\bar{z}$}

The relation of a field wave to a magnetization wave in a thin horizontal plate has been developed in para 3.1. Since in the models described in para 4.3. the inclination has been assumed to be $I=90^{\circ}$ equation (15) can be rewritten for $\Delta Z$ as follows

$$
|\Delta Z(\bar{z}, \bar{z}, \infty)|=2 \pi J_{m}{ }^{(k)}
$$

This is the amplitude of the field wave in $\bar{z}$ if the magnetization wave with the amplitude $J_{m}{ }^{(k)}$ is present in the entire half space $z>\bar{z}$. If this magnetization is present only in the thin horizontal plate between $\bar{z}$ and $\bar{z}+\Delta z$ the field wave in the plane $\bar{z}$ according to (18) has the amplitude

$$
|\Delta Z(\bar{z}, \bar{z}, \bar{z}+\Delta z)|=J_{m}{ }^{(k)} \cdot\left(4 \pi^{2} \sqrt{k^{2}+m^{2}} / \Lambda\right) \cdot \Delta z .
$$

For the further treatment in this appendix this equation is rewritten with the following symbols

$$
d K_{m}^{(k)}(z)=J_{m}{ }^{(k)}(z) \cdot\left(4 \pi^{2} \sqrt{k^{2}+m^{2}} / \Lambda\right) \cdot d z .
$$

Now the field wave $k, m$ must be continued upward from the plane $z$ to $\bar{z}$ which is the upper boundary plane of the entire model :

$$
d K_{m}{ }^{(k)}(\bar{z})=J_{m}{ }^{(k)}(z) \cdot\left(4 \pi^{2} \sqrt{k^{2}+m^{2}} / \Lambda\right) \cdot \exp \left(2 \pi(\bar{z}-z) \sqrt{k^{2}+m^{2}} / \Lambda\right) \cdot d z
$$

Inserting (66a) in (71) we yield 


$$
\begin{gathered}
d K_{m}{ }^{(k)}(\bar{z})=\frac{-\sqrt{k^{2}+m^{2}}}{\Lambda k m} \sum_{\mu=1}^{g(\nu)} \sum_{\lambda=1}^{l(\mu, \nu)} J(\lambda, \mu, z) D_{1}\left(x_{\lambda \mu_{\nu}}\right) D_{2}\left(y_{\mu_{\nu}}\right) . \\
\exp \left(2 \pi(\bar{z}-z) \sqrt{k^{2}+m^{2}} / \Lambda\right) \cdot d z
\end{gathered}
$$

\section{The integral over the entire vertical extension of the model}

Considering that the model is finite in $z$-direction extending between the planes $z=z_{0}$ and $z=z_{0}+T, T$ denoting the thickness of the model,this integral is

$$
\begin{gathered}
K_{m}{ }^{(k)}(\bar{z})=\frac{-\sqrt{k^{2}+m^{2}}}{\Lambda k m} \int_{\bar{z}}^{\bar{z}+T} \sum_{\mu=1}^{g(\nu)} \sum_{\lambda=1}^{l(\mu, \nu)} J(\lambda, \mu, z) D_{1}\left(x_{\lambda \mu_{\nu}}\right) D_{2}\left(y_{\mu_{\nu}}\right) \cdot \\
\exp \left(2 \pi(\bar{z}-z) \sqrt{k^{2}+m^{2}} / \Lambda\right) d z .
\end{gathered}
$$

$J(\lambda, \mu, z)$ is constant in every layer $\nu$ for all values of $z$. So this integral can be written as the sum

$$
K_{m}^{(k)}\left(z_{0}\right)=\frac{1}{2 \pi k m} \sum_{\nu=1}^{h} \sum_{\mu=1}^{g(\nu)} \sum_{\lambda=1}^{l(\mu, \nu)} J(\lambda, \mu, \nu) D_{1}\left(x_{\lambda \mu_{\nu}}\right) D_{2}\left(y_{\mu_{\nu}}\right) \cdot D_{3}\left(z_{\nu}\right)
$$

Here $h$ signifies the number of the layers between $\bar{z}$ and $\bar{z}+T$, and $J(\lambda, \mu, \nu)$ the magnetization of the $\lambda$ th volume part in the $\mu$ th row in the $\nu$ th layer. $D_{3}\left(z_{\nu}\right)$ is an abbreviation for

$$
D_{3}\left(z_{\nu}\right)=\exp \left(2 \pi\left(z_{0}-z_{\nu}\right) \sqrt{k^{2}+m^{2} / \Lambda}\right)-\exp \left(2 \pi\left(z_{0}-z_{\nu-1}\right) \sqrt{k^{2}+m^{2}} / \Lambda\right)
$$

$\nu=1,2, \ldots, h$. If $\nu=1$, the difference $\bar{z}-z_{0}$ will occur in the second term. According to the definitions (28) both these values denote the $z$-coordinate of the upper boundary of the model:

$$
z_{0}=\bar{z} \text {. }
$$

The two-dimensional amplitude spectrum of the field in $z_{0}$ is derived from (74) by multiplying $K_{m}{ }^{(k)}(\bar{z})$ by its conjugate complex value $K_{-m}{ }^{(-k)}(\bar{z})$. The product of these two threefold sums are written as a sixfold sum where the indices $\lambda, \mu, \nu$ of the conjugate complex terms are marked by $\lambda^{\prime}, \mu^{\prime}, \nu^{\prime}$; the corresponding terms $\mathrm{D}_{1}, \mathrm{D}_{2}, \mathrm{D}_{3}$ by $\mathrm{D}_{1}{ }^{*}, \mathrm{D}_{2}{ }^{*}, \mathrm{D}_{3} *$.

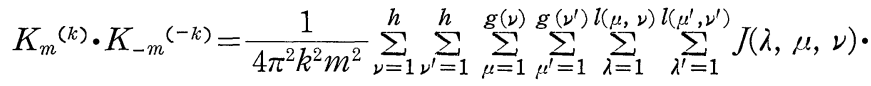

$$
\begin{aligned}
& J\left(\lambda^{\prime}, \mu^{\prime}, \nu^{\prime}\right) D_{1}\left(x_{\lambda \mu_{\nu} \nu}\right) D_{1} *\left(x_{\lambda^{\prime} \mu^{\prime} \nu^{\prime}}\right) D_{2}\left(y_{\mu_{\nu}}\right) D_{2} *\left(y_{\mu^{\prime} \nu^{\prime}}\right) D_{3}\left(z_{\nu}\right) D_{3} *\left(z_{\nu^{\prime}}\right) \text {. }
\end{aligned}
$$

\section{Expectancy value for $K_{m}{ }^{(k)} \cdot K_{-m}{ }^{(-k)}$}

To calculate the expectancy value of $K_{m}{ }^{(k)} \cdot K_{-m}{ }^{(-k)}$ some assumptions must be made which shall be given at those places where they are needed.

For the evaluation of the two innermost sums over $\lambda$ and $\lambda^{\prime}$ the assumption is made that for all fixed numbers $\mu_{1}, \nu_{1}$ the sequences $J\left(\lambda, \mu_{1}, \nu_{1}\right) \cdot \operatorname{Re}\left(D_{1}\left(x_{\lambda \mu_{\nu}}\right)\right)$ and $J\left(\lambda, \mu_{1}, \nu_{1}\right) \cdot \operatorname{Im}\left(D_{1}\left(x_{\lambda \mu_{\nu}}\right)\right)$ are distributed like the results of two-dimensional random walks in the plane of the complex figures showing no preferred direction. This assumption involves that there is no correlation between the differences of the sin- and cos-values of the partial waves at the boundaries of the volume elements and the magnetization of those elements.

The expectancy value of the two innermost sums is

$$
E\left\{\sum_{\lambda=1}^{l(\mu, \nu) l\left(\mu^{\prime}, \nu^{\prime}\right)} \sum_{\lambda^{\prime}=1} J(\lambda, \mu, \nu) J\left(\lambda^{\prime}, \mu^{\prime}, \nu^{\prime}\right) D_{1}\left(x_{\lambda \mu_{\nu}}\right) D_{1} *\left(x_{\lambda^{\prime} \mu^{\prime} \nu^{\prime}}\right)\right\}
$$


If $\mu=\mu^{\prime}$ and $\nu=\nu^{\prime}(78)$ can be rewritten as follows:

$$
\begin{aligned}
(78) & =E\left\{\sum_{\lambda=1}^{l(\mu, \nu)} J^{2}(\lambda, \mu, \nu) D_{1}\left(x_{\lambda \mu_{\nu}}\right) D_{1} *\left(x_{\lambda \mu_{\nu}}\right)\right\} \\
& +E\left\{\sum_{\lambda=1}^{l(\mu, \nu) l\left(\mu, \nu^{\prime}\right)} \sum_{\substack{\lambda^{\prime}=1 \\
\lambda^{\prime} \neq \lambda}} J(\lambda, \mu, \nu) J\left(\lambda^{\prime}, \mu^{\prime}, \nu^{\prime}\right) D_{1}\left(x_{\lambda \mu_{\nu}}\right) D_{1} *\left(x_{\lambda^{\prime} \mu^{\prime} \nu^{\prime}}\right)\right\}
\end{aligned}
$$

The double sum over the products with unequal indices can be rewritten:

$$
\begin{gathered}
E\left\{\sum _ { \substack { \lambda = 1 \\
l ( \mu , \nu ) l ( \mu , ^ { \prime } \nu ^ { \prime } ) } } \sum _ { \substack { \lambda ^ { \prime } = 1 \\
\lambda ^ { \prime } \neq \lambda } } J ( \lambda , \mu , \nu ) \left(\operatorname{Re}\left(D_{1}\left(x_{\lambda \mu_{\nu}}\right)\right)+i \cdot \operatorname{Im}\left(D_{1}\left(x_{\lambda \mu_{\nu}}\right)\right) \cdot\right.\right. \\
J\left(\lambda^{\prime}, \mu^{\prime}, \nu^{\prime}\right)\left(\operatorname{Re}\left(D_{1} *\left(x_{\lambda^{\prime} \mu^{\prime} \nu^{\prime}}\right)\right)+i \cdot \operatorname{Im}\left(D_{1} *\left(x_{\lambda^{\prime} \mu^{\prime} \nu^{\prime}}\right)\right)\right\}
\end{gathered}
$$

Regarding the assumptions made above and the fact that the average values of $J(\lambda, \mu, \nu)$ and those of $D_{1}\left(x_{2 \mu_{\nu}}\right)$ are vanishing the expectancy of (80) turns out to be zero.

If

$$
\begin{array}{r}
\mu \neq \mu^{\prime} \text { and } \nu \neq \nu^{\prime} \text { or } \nu \neq \nu^{\prime} \text { and } \mu \neq \mu^{\prime} \\
\text { or } \nu=\nu^{\prime}
\end{array}
$$

we yield from (78) only one double sum:

$$
\begin{gathered}
E\left\{\sum_{\lambda=1}^{l(\mu, \nu)} \sum_{\lambda^{\prime}=1}^{l\left(\mu^{\prime}, \nu^{\prime}\right)} J(\lambda, \mu, \nu)\left[\operatorname{Re}\left(D_{1}\left(x_{\lambda \mu_{\nu}}\right)\right)+i \cdot \operatorname{Im}\left(D_{1}\left(x_{\lambda \mu_{\nu}}\right)\right)\right] .\right. \\
\left.J\left(\lambda^{\prime}, \mu^{\prime}, \nu^{\prime}\right)\left[\operatorname{Re}\left(D_{1} *\left(x_{\lambda^{\prime} \mu^{\prime} \nu^{\prime}}\right)\right)+i \cdot \operatorname{Im}\left(D_{1} *\left(x_{\lambda^{\prime} \mu^{\prime} \nu^{\prime}}\right)\right)\right]\right\}
\end{gathered}
$$

Under the assumption that there is no correlation between the sequences $J(\lambda, \mu, \nu)$. $\left.\operatorname{Re}\left(D_{1}\left(x_{\lambda \mu_{\nu}}\right)\right), J\left(\lambda^{\prime}, \mu^{\prime}, \nu^{\prime}\right) \cdot \operatorname{Re}\left(D_{1} *\left(x_{\lambda^{\prime} \mu^{\prime} \nu^{\prime}}\right)\right), J(\lambda, \mu, \nu) \cdot \operatorname{Im}\left(x_{\lambda \mu_{\nu} \nu}\right)\right), J\left(\lambda^{\prime}, \mu^{\prime}, \nu^{\prime}\right) \cdot \operatorname{Im}\left(D_{1}\left(x_{\lambda^{\prime} \mu^{\prime} \nu^{\prime}}\right)\right)$ the expectancy (82) under the condition (81) will vanish. Thus only the single sum of (79) remains unequal zero. Its value is

$$
(78)={\overline{J^{2}}}^{\lambda}(\mu, \nu) \sum_{\lambda=1}^{l(\mu, \nu)} D_{1}\left(x_{\lambda \mu_{\nu}}\right) D_{1}^{*}\left(x_{\lambda \mu_{\nu}}\right)
$$

Here the sign $-\lambda$ denotes the average value of $J^{2}$ taken over all values $\lambda$. The result is still dependent on $\mu, \nu$. The products $D_{1} D_{1} *$ are according to (67)

$$
\begin{aligned}
& D_{1}\left(x_{\lambda \mu_{\nu}}\right) D_{1} *\left(x_{\lambda \mu_{\nu}}\right)=\left(\exp \left(-2 \pi i \cdot k x_{\lambda \mu_{\nu}} / \Lambda\right)-\exp \left(-2 \pi i \cdot k x_{\lambda-1 \mu_{\nu}} / \Lambda\right)\right) \cdot \\
& \quad\left(\exp \left(2 \pi i \cdot k x_{\lambda \mu_{\nu}} / \Lambda\right)-\exp \left(2 \pi i k x_{\lambda-1 \mu_{\nu}} / \Lambda\right)\right)=2-2 \cos \left(2 \pi k\left(x_{\lambda \mu_{\nu}}-x_{\lambda-1 \mu_{\nu}}\right) / \Lambda\right) .
\end{aligned}
$$

The difference $x_{\lambda \mu_{\nu}}-x_{\lambda-1 \mu_{\nu}}$ is distributed uniformly according to the definition of the model, between $L_{1}$ and $L_{2}$ without dependency on $\mu$ and $\nu$. Hence we find:

$$
\begin{gathered}
E\left[2-2 \cdot \cos \left(2 \pi k\left(x_{\lambda \mu_{\nu}}-x_{\lambda-1 \mu_{\nu}}\right) / \Lambda\right]=2-\frac{2}{L_{2}-L_{1}} \int_{L_{1}}^{L_{2}} \cos (2 \pi k x / \Lambda) d x\right. \\
=2-\frac{\Lambda}{\pi k\left(L_{2}-L_{1}\right)}\left(\sin \left(2 \pi k L_{2} / \Lambda\right)-\sin \left(2 \pi k L_{1} / \Lambda\right)\right) .
\end{gathered}
$$

Inserting (85) in (83) we finally yield the expectancy of the two innermost sums of (77): 


$$
\begin{gathered}
(78)=\bar{J}^{2}(\mu, \nu) \cdot l(\mu, \nu)\left(2-\frac{\Lambda}{\pi k\left(L_{2}-L_{1}\right)} \cdot\right. \\
\left.\left(\sin \left(2 \pi k L_{2} / \Lambda\right)-\sin \left(2 \pi k L_{1} / \Lambda\right)\right)\right)
\end{gathered}
$$

Calculating the next four sums over $\mu, \mu^{\prime}, \nu$ and $\nu^{\prime}$ we remember that the expectancy of the two inner sums of (77) is different from zero only if $\mu=\mu^{\prime}$ and $\nu=\nu^{\prime}$. So in analogy to the foregoing procedure the expectancy value of the four inner sums turns out to be

$$
\begin{aligned}
{\overline{\bar{J}^{2}}}^{\mu}(\nu) g(\nu) & \overline{l(\nu)} \cdot \\
& {\left[2-\frac{\Lambda}{\pi k\left(L_{2}-L_{1}\right)}\left(\sin \left(2 \pi k L_{2} / \Lambda\right)-\sin \left(2 \pi k L_{1} / \Lambda\right)\right)\right] . } \\
& {\left[2-\frac{\Lambda}{\pi m\left(M_{2}-M_{1}\right)}\left(\sin \left(2 \pi m M_{2} / \Lambda\right)-\sin \left(2 \pi m M_{1} / \Lambda\right)\right)\right] }
\end{aligned}
$$

Considering the last two sums over $\nu$ and $\nu^{\prime}$ we obtain

$$
\begin{aligned}
& E\left(K_{m}{ }^{(k)} K_{-m}{ }^{(-k)}\right)=\frac{1}{\pi^{2} k^{2} m^{2}} \overline{{\overline{\bar{J}^{2}}}^{\mu^{\nu}}} \overline{\bar{l}^{\mu^{\nu}}} \bar{g} \cdot \\
& \left(1-\frac{\Lambda}{2 \pi k\left(L_{2}-L_{1}\right)}\left(\sin \left(2 \pi k L_{2} / \Lambda\right)-\sin \left(2 \pi k L_{1} / \Lambda\right)\right) .\right. \\
& \left(1-\frac{\Lambda}{2 \pi m\left(M_{2}-M_{1}\right)}\left(\sin \left(2 \pi m M_{2} / \Lambda\right)-\sin \left(2 \pi m M_{1} / \Lambda\right)\right)\right. \text {. } \\
& \sum_{\nu=1}^{h} D_{3}\left(z_{\nu}\right) D_{3} *\left(z_{\nu}\right)
\end{aligned}
$$

The expectancy value of the sum over the terms with $\nu \neq \nu^{\prime}$ is zero because under this condition the expectancy of the inner sums is vanishing (see (81) and (82)).

$D_{3}\left(z_{\nu}\right)$ is a real quantity. Thus its product with its conjugate complex value is nothing but its square, and the last sum involved in (88) has the value

$$
\begin{aligned}
& \sum_{\nu=1}^{h}\left(D_{3}\left(z_{\nu}\right)\right)^{2}=\sum_{\nu=1}^{h}\left(\exp \left(2 \pi\left(z_{0}-z_{\nu}\right) \sqrt{k^{2}+m^{2}} / \Lambda\right)\right. \\
& \left.\quad-\exp \left(2 \pi\left(z_{0}-z_{\nu-1}\right) \sqrt{k^{2}+m^{2}} / \Lambda\right)\right)^{2} \\
& \quad=\sum_{\nu=1}^{h} \exp \left(4 \pi\left(z_{0}-z_{\nu-1}\right) \sqrt{k^{2}+m^{2}} / \Lambda\right)\left(1-\exp \left(2 \pi\left(z_{\nu-1}-z_{\nu}\right) \sqrt{k^{2}+m^{2} / \Lambda}\right)\right)^{2}
\end{aligned}
$$

The difference $z_{\nu}-z_{\nu-1}$ is the thickhess $N$ of the layers of the model. This is constant for all layers :

$$
\begin{gathered}
z_{\nu}-z_{\nu-1}=N, z_{\nu-1}-z_{0}=(\nu-1) N ; \\
z_{h}-z_{0}=h N=T \quad(\text { see }(28))
\end{gathered}
$$

is the finite thickness of the entire model. Hence (89) can be written

$$
\begin{gathered}
(89)=\left(1-\exp \left(-2 \pi N \sqrt{k^{2}+m^{2}} / \Lambda\right)\right)^{2} \\
\sum_{\nu=1}^{h} \exp \left(-4 \pi(\nu-1) N \sqrt{k^{2}+m^{2}} / \Lambda\right)
\end{gathered}
$$

The sum involved here is a geometric series: 


$$
\sum_{\nu=1}^{h} \exp \left(-4 \pi(\nu-1) N \sqrt{k^{2}+m^{2}} / \Lambda\right)=\frac{1-\exp \left(-4 \pi T \sqrt{k^{2}+m^{2}} / \Lambda\right)}{1-\exp \left(-4 \pi N \sqrt{k^{2}+m^{2}} / \Lambda\right)}
$$

Inserting (92) into (89) and this again into (88) we finally obtain

$$
\begin{aligned}
E\left(K_{m}{ }^{(k)} K_{-m}{ }^{(-k)}\right)=\frac{1}{\pi^{2} k^{2} m^{2}} \cdot \overline{J^{2}} \cdot \frac{4 \Lambda^{2}}{\left(L_{2}+L_{1}\right)\left(M_{2}+M_{1}\right)} \cdot & \\
& \left(1-\frac{\Lambda}{2 \pi k\left(L_{2}-L_{1}\right)}\left(\sin \left(2 \pi k L_{2} / \Lambda\right)-\sin \left(2 \pi k L_{1} / \Lambda\right)\right) \cdot\right. \\
& \left(1-\frac{A}{2 \pi m\left(M_{2}-M_{1}\right)}\left(\sin \left(2 \pi m M_{2} / \Lambda\right)-\sin \left(2 \pi m M_{1} / \Lambda\right)\right) \cdot\right. \\
& \frac{1-\exp \left(-2 \pi N \sqrt{k^{2}+m^{2}} / \Lambda\right)}{1+\exp \left(-2 \pi N \sqrt{k^{2}+m^{2}} / \Lambda\right)}\left(1-\exp \left(-4 \pi T \sqrt{k^{2}+m^{2}} / \Lambda\right)\right.
\end{aligned}
$$

Here the standard deviation of the magnetization values of all volume parts is denoted by $\overline{J^{2}}$; the average number $\overline{\bar{l}}^{\mu^{\nu}}$ of volume parts in one row and the average number of rows $\bar{g}^{\nu}$ in one interval $\langle 0, A\rangle$ are replaced by the quotient of the period and the average length and width of the parts

$$
\overline{\bar{l}}^{\mu^{\nu}}=\frac{2 \Lambda}{L_{2}+L_{1}} \quad \bar{g}^{\nu}=\frac{2 \Lambda}{M_{2}+M_{1}} .
$$

Equation (93) is the expectancy value of the two-dimensional amplitude spectrum of the field in the plane $z=z_{0}$ which is the upper surface of the given model. The spectra in higher levels $z_{e}$ above the model are obtained by multiplying (93) by the factor $\exp \left(2 \pi\left(z_{e}-\bar{z}\right)\right.$ $\left.\sqrt{k^{2}+m^{2}} / \Lambda\right)$.

\section{Appendix 3}

The expectancy of the profile spectrum in the quasi-nonperiodical short interval $A / 3$.

It is explained in para 4.3. that the function

$$
\sum_{N=-R / 2}^{R / 2} K_{N} \exp (2 \pi i N \rho / R)
$$

which has the period $d \cdot R=\Lambda$ is analyzed in the short interval

$$
\lambda=\Lambda / 3=d \cdot R / 3=d \cdot r ; r=R / 3,
$$

leading to the coefficients $k_{n}$. Since the function (33) with respect to the short interval $\lambda$ is quasi-nonperiodic the coefficients $k_{n}$ are comparable to the coefficients obtained from the nonperiodic profiles measured in the field.

The calculation of the coefficients $k_{n}$ as functions of the $K_{N}$ starts from equation (36) which is repeated here

$$
\begin{aligned}
k_{n}=\frac{1}{r} \sum_{\rho=0}^{r-1} \sum_{N=-R / 2}^{R / 2} \exp (-2 \pi i n \rho / r) . \\
K_{n}\left(\exp (2 \pi i N \rho / 3 r)-\frac{\rho}{r}(\exp (2 \pi i N / 3)-1)\right) .
\end{aligned}
$$

We rewrite this by exchange of the summations 


$$
\begin{gathered}
k_{n}=\frac{1}{r} \sum_{N=-R / 2}^{R / 2} K_{N} \sum_{\rho=0}^{r-1}[\exp (2 \pi i(N-3 n) / 3 r)]^{\rho} \\
-\frac{1}{r}(\exp (2 \pi i N / 3)-1) \rho[\exp (-2 \pi i n / r)]^{\rho}
\end{gathered}
$$

The inner sum can be split into two sums of the form

$$
\sum_{\rho=0}^{r-1} \mathrm{a}^{\rho} \text { and } b \sum_{\rho=0}^{r-1} \rho c^{\rho}, \text { respectively. }
$$

The first one has the value

$$
\sum_{\rho=0}^{r-1} a^{\rho}=\frac{1-a^{r}}{1-a} .
$$

The second one is calculated in the form of a double sum :

$$
b \sum_{\rho=0}^{r-1} \rho c^{\rho}=b \sum_{\sigma=1}^{r-1} \sum_{\rho=\sigma}^{r-1} c^{\rho}=b \sum_{\sigma=1}^{r-1} \frac{c^{\sigma}-c^{r}}{1-c}=b \cdot \frac{c-r c^{r}+(r-1) c^{r+1}}{(1-c)^{2}} .
$$

Using these formulas and considering that $\exp (2 \pi i k)=1$ if $k$ is an integer we find the value of (36):

$$
\begin{aligned}
k_{n}= & \frac{1}{r} \sum_{\substack{N=-R / 2 \\
N \neq 3 n}}^{R / 2} K_{N}(1-\exp (2 \pi i N / 3) . \\
& {\left[\frac{1}{1-\exp (2 \pi i(N-3 n) / 3 r)}-\frac{1}{1-\exp (-2 \pi i n / r)}\right]+K_{3 n} . }
\end{aligned}
$$

Now the expectancy of the real amplitude $c_{n}$ is

$$
E\left(c_{n}\right)=\sqrt{E\left(c_{n}^{2}\right)}=\sqrt{E\left(4 k_{n} k_{-n}\right)} .
$$

This can be seen from equation (26). Hence, in order to calculate this expectancy we have to multiply $k_{n}$ by $k_{-i \imath}$. This is a sum of $R^{2}$ products. But only the products of the terms with the index $N_{1}$ in the sum $k_{n}$ and the terms with the index $-N_{1}$ in the sum $k_{-n}$ will always have a positive real value. All other products are complex with an arbitrary phase wh:ch is not correlated to their magnitude. Hence only the expectancy of the former products is finite whereas the expectancy of the latter products vanishes. We find

$$
\begin{aligned}
& E\left(c^{2}\right)=4 E\left(k_{n} k_{-n}\right)=E\left\{4 K_{3 n} K_{-3 n}+\right. \\
& \quad+\frac{4}{r^{2}} \sum_{\substack{N=-R_{N / 2} \\
N=3 n}}^{R / 2} K_{N} K_{-N}(1-\exp (2 \pi i N / 3))(1-\exp (-2 \pi i N / 3)) \\
& \\
& \quad\left[\frac{1}{1-\exp (2 \pi i(N-3 n) / 3 r)}+\frac{1}{1-\exp (2 \pi i(-3 n) / 3 r)}\right] \\
& \left.\left.\quad \frac{1}{1-\exp (-2 \pi i(N-3 n) / 3 r)}+\frac{1}{1-\exp (-2 \pi i(-3 n) / 3 r)}\right]\right\}
\end{aligned}
$$

A lengthy but elementary calculation leads to

$$
E\left(c_{n}^{2}\right)=E\left(C_{3 n}^{2}\right)+\frac{1}{r^{2}} \sum_{\substack{N=1 \\ N \neq 3 n}}^{R / 2} E\left(C_{N}^{2}\right)\left[\frac{\sin (\pi N / 3) \cdot \sin (\pi N / 3 r)}{\sin (\pi n / r)}\right]^{2} .
$$




$$
\left\{\left[\frac{1}{\sin (\pi(N-3 n) / 3 r)}\right]^{2}+\left[\frac{1}{\sin (\pi(N+3 n) / 3 r)}\right]^{2}\right\}
$$

Here $C_{N}$ denotes the real coefficient $C_{N}=4 K_{N} K_{-N}$. The summation in (100) is extended from 1 to $R / 2$ only corresponding to two terms in the last bracket \{ \} of the sum; the sum over $-R / 2$ to $R / 2$ would have the first term only. The term involving $C_{0}$ vanishes.

Equation (100) is identical with (37) rewritten in a form to be practized in a computer program.

\section{References}

Angenheister, G. Nachr. Akad. Wiss Göttingen, mathem.-phys. Klasse, IIa, Nr.9. (1956)

Angenheister, G. personal communication. (1957)

Baranov, V. Geophys. 22, 359. (1957)

Bentz, A. Lehrbuch der Angewandten Geologie, Stuttgart (1961)

Chastenet de Gery, J. \& Naudy, H. Geophys. prospect. 5, 421. (1957)

Dohr, G. Zeitschr. Geophys. 25/6, 280. (1959)

Giret, R. \& Naudy, H. Papers of the Sixth WPC in Frankfurt/Main, Section I-Paper 15-PD 4, France. (1963)

Nagata, T. Bull. Earthqu. Res. Inst. 16, 550. (1938)

Peters, L.J. Geophys. 11,1. (1949)

Rayleigh Phil. Mag. Ser. 5, Vol. 10. (1880)

Reich, H. Geol. Jb. 64, 243. (1950)

Schulze, G.A. \& Förtsch, O. Geol Jb. 64, 205. (1950)

Tsuboi \& Fuchida Bull. Earthqu. Res. Inst. 15, 636. (1937)

Tsuboi \& Fuchida Bull. Earthqu. Res. Inst. 16, 273 (1938)

Turkowsky Diplomarbeit Univ. München-unpublished (1962)

Vacquier, V, Steenland, N, Henderson, R.G., Zietz, I. Geol. Soc. Amer. Memoir 47. (1951)

Vestine, E.H., \& Davids, N. Terr. Mag. 50. 1. (1945) 